Article

\title{
Economizing the Uneconomic: Markets for Reliable, Sustainable, and Price Efficient Electricity
}

\author{
Mohammad Rasouli ${ }^{1, *,+(\mathbb{D})}$ and Demosthenis Teneketzis ${ }^{2}$ \\ 1 Civil and Environmental Engineering Department, Stanford, Palo Alto, CA 94305, USA \\ 2 Electrical and Computer Engineering Department, University of Michigan, Ann Arbor, MI 48109, USA; \\ teneket@umich.edu \\ * Correspondence: rasoulim@stanford.edu \\ † 235 Embarcadero Road, Palo Alto, CA 94301, USA.
}

Citation: Rasouli, M.; Teneketzis, D. Economizing the Uneconomic: Markets for Reliable, Sustainable, and Price Efficient Electricity.

Sustainability 2021, 13, 4197. https:// doi.org/10.3390/su13084197

Academic Editor: Mohammad Jafari

Received: 20 February 2021

Accepted: 21 March 2021

Published: 9 April 2021

Publisher's Note: MDPI stays neutral with regard to jurisdictional claims in published maps and institutional affiliations.

Copyright: () 2021 by the authors. Licensee MDPI, Basel, Switzerland. This article is an open access article distributed under the terms and conditions of the Creative Commons Attribution (CC BY) license (https:// creativecommons.org/licenses/by/ $4.0 /)$.

\begin{abstract}
Current electricity markets do not efficiently achieve policy targets i.e., sustainability, reliability, and price efficiency. Thus, there are debates on how to achieve these targets by using either market mechanisms e.g., carbon and capacity markets, or non-market mechanisms such as offer-caps, price-caps, and market-monitoring. At the same time, major industry changes including demand response management technologies and large scale batteries bring more elasticity to demand; such changes will impact the methodology needed to achieve the above mentioned targets. This work provides market solutions that capture all three policy targets simultaneously and take into account the above-mentioned industry changes. The proposed solutions are based on: (i) a model of electricity markets that captures all the above mentioned electricity policy targets; (ii) mechanism design and the development of a framework for design of efficient auctions with constraints (individual, joint homogeneous, and joint non-homogeneous). The results show that, within the context of the proposed model, all policy targets can be achieved efficiently by separate capacity and carbon markets in addition to efficient spot markets. The results also highlight that all three policy targets can be achieved without any offer-cap, price-cap, or market monitoring. Thus, within the context of the proposed model, they provide clear answers to the above-mentioned policy debates.
\end{abstract}

Keywords: electricity policy; electricity market; mechanism design; auctions with constraints

\section{Introduction}

\subsection{Motivation}

Electricity policies in most countries aim at providing reliable and sustainable electricity with an efficient price to customers [1]. Reliability refers to meeting the uncertain demand almost at all times. Sustainability primarily refers to overcoming global warming (sustainability solutions are subject to being economically profitable as emphasized in new studies e.g., [2]). Price efficiency (affordability) refers to non-discriminatory price equal to the marginal cost of production for all participants. System operators translate reliability target into different factors e.g., planning reserve requirement, $n-1$ criterion, interconnection frequency response, element availability percentage, etc.). Modeling all the reliability constraints will make the model and presentation complicated. Hence, in this study, we consider only the planning reserve requirement for reliability (whenever reliability is referred to in this work, it means meeting the planning reserve requirement), that is, the total capacity should exceed a threshold to ensure uncertain supply can meet the uncertain demand at almost all outcomes (depending on the definition used by system operator this could be one in ten year blackout or alternative more tight constraints); planning reserve requirement was chosen because it is at the center of the debate for the need for capacity markets as it is about sufficient capacity at the time of planning that operational constraints. The sustainability target is translated into the carbon cap, which is a cap for total carbon emission (note that the calculation of the carbon cap is subject to international 
agreements for global warming limits and advancements in other economically viable solutions to reduce carbon emission particularly carbon sequestration [3-5]). Planning reserve requirements and carbon caps are uneconomic constraints (public constraints) because generation companies (GenCos) do not have incentives to meet them unless appropriate taxes/subsidies are in place. Addressing the policy targets for the electricity industry should be consistent with the spirit of the restructured industry i.e., should be based on market competition, incentives, and minimum regulations.

Before restructuring, the system operator could set the selling price, the investment level, and the technology in-use and in this way could ensure price efficiency, reliability, and sustainability. After restructuring however, the current electricity markets suffer from high prices above marginal cost of production and scarce resources (capacity underinvestment); for example in the California 2000 electricity crisis, market imperfections such as market power and missing money problems (investors not collecting their cost of investment) led to very high prices [6]. Moreover, post-restructuring mechanisms used for reducing carbon emission, such as carbon tax, carbon markets, or renewable feed-in tariffs, are inefficient and costly $[7,8]$. Especially, this inefficiency increases with a larger share of renewables $[9,10]$.

There are significant debates on the approaches to the correct solutions of these problems: The first is centered around the need for non-market mechanisms like price-cap, offer-cap, and market-monitoring so as to achieve efficient prices. The second is about the need for mechanisms with direct capacity incentives, like capacity subsidy or capacity market, so as to achieve the reliability target; this approach is referred to as the energy-andcapacity solution in contrast to the energy-only solution. Even without planning reserve requirements for reliability, some references argue that capacity mechanisms are required for price efficiency because GenCos' investment will not be socially optimal due to their market power [11,12]. The third is with regard to carbon tax that is introduced so as to achieve sustainability target and refers to the need for the system operator to set the carbon tax (i.e., price policy) or the possibility of finding the efficient carbon tax by carbon markets (i.e., quantity policy).

While the above debates are still ongoing, the electricity industry is under major technological changes, with the most prominent ones being smart grids with demand response management (DRM), electricity storage both in the form of large scale batteries or small batteries (such as behind-the-meter batteries or electrical vehicles), and renewable technologies. First, smart grids facilitate response of the demand to the prices. This facility is used by DRM programs for demand shaping, demand shifting, and load shedding [13]. As a consequence, the demand in the wholesale market will be elastic to the prices. Second, electricity storage allows for a mismatch between supply and demand by charging (discharging) the surplus (deficit) to (from) the storage. Depending on the electricity prices, a battery can act like an elastic demand or a producer in the market [14]. Third, renewables discourage investment on electricity in two ways: (i) their low marginal cost of production reduces the prices in the wholesale (spot) market and therefore makes it harder for both renewable and conventional utilities to recover their investment costs [15]; (ii) renewables' high uncertainty makes them a risky investment $[16,17]$. On the other hand, lower carbon emission of renewables can make them an interesting investment for producers if appropriate taxes/subsidies are in place [9]. Consequently, the available studies in the literature should be updated by considering the technological changes in the industry.

The existing studies of electricity policies are limited to addressing reliability, sustainability, and price efficiency separately and in isolation; however, these issues are in fact intertwined [18]. For example, any changes in the wholesale spot market for affordable prices, like changes in price caps, can impact reliability by changing investment incentives; reliability subsidies, like capacity investment subsidy, work against carbon taxation so as to reduce the capacity of conventional electricity; renewables subsidies result in more intermittent wind and solar resources and reduce reliability, etc. In fact, the interdisciplinary implications of the renewable policies are extensively shown in the literature; for 
example, Reference [19] shows corporate innovation and performance linked to green practices, References [20,21] study green incentives for farmers decisions, and [22] studies incentives for biorefinery. Therefore, the above-stated policy targets should be studied together. To the best of the authors' knowledge, Reference [23] is the only study that considers all three policy targets together but it is limited to showing the shortcomings of the current markets by analyzing their performance. The second shortcoming of the literature is that majority of the existing studies analyze the current markets in order to evaluate their performance with respect to reliability, sustainability, and price efficiency. However, in the authors' opinion, the correct approach for addressing these problems is a design approach starting from the desired policy targets.

This work provides market solutions that capture all three policy targets simultaneously and take into account the above-mentioned industry changes. The proposed solutions are based on: (i) a model of electricity markets that captures all the above mentioned electricity policy targets; (ii) mechanism design and the development of a framework for design of efficient auctions with constraints (individual, joint homogeneous, and joint non-homogeneous). The results show that within the context of the proposed model, all policy targets can be achieved efficiently by separate capacity and carbon markets in addition to efficient spot markets. They also highlight that all three policy targets can be achieved without any offer-cap, price-cap, or market monitoring. Thus, within the context of the proposed model, they provide clear answers to the above-mentioned policy debates.

Notation: Bold letters refer to random variables; the realizations of random variables are denoted by the corresponding non-bold letters. Capital letters refer to both sets and vectors. Such a notation is not going to create any confusion because it will be always clear from the context of the text where capital symbols appear. For convenience this paper uses the notation $a_{x: y}$ to refer to $\left\{a_{x}, a_{x+1}, \ldots, a_{y-1}, a_{y}\right\}, a_{x: y, v}$ to refer to $\left\{a_{x, v}, \ldots, a_{y, v}\right\}$, and $a_{x: y, v: w}$ to refer to $\left\{a_{x: y, v}, a_{x: y, v+1}, \ldots, a_{x: y, w}\right\}$.

\subsection{Objective}

The objective of this study is to provide a response to the debate about efficiency of market mechanisms vs non-market mechanisms for achieving electricity policy targets stated in Section 1.1, namely, reliability, sustainability, and price efficiency while considering the effect of the new technologies, particularly demand elasticity due to demand response management programs and electricity storage. To this end, it design markets that achieve all of the above-mentioned three electricity policy targets simultaneously, while considering the demand elasticity due to new technologies.

\subsection{Approach to Achieving the Objectives}

To achieve the objective, this work proceeds in two steps. First, it proposes a model of both the electricity industry, considering emerging electricity technological changes, and the policy targets. Second, for the proposed model, using ideas from auction theory and Nash implementation, it designs markets/mechanisms that achieve sustainability, reliability, and price efficiency at all Nash equilibria of the games induced by the mechanisms.

We start by modeling an oligopoly of strategic GenCos with symmetric information that interacts over a long horizon. The demand for electricity is elastic but non-strategic (i.e., demand is a price-taker). This means that the system operator wants to auction the demand among the strategic GenCos. In this model, each GenCo's electricity generation is constrained by its capacity. Next, the policy targets is added to the model. The reliability target is modeled by a constraint requiring that the sum of the GenCos' capacities exceed the planning reserve requirement. The sustainability target is modeled by a constraint requiring that the sum of the carbon emissions is below the carbon cap. Price efficiency is modeled as a desired feature of the electricity market at equilibrium. This stylized model captures, the major characteristics of the restructured electricity industry that can highlight the innovations presented here for studying the policy targets. 
To design electricity markets/mechanisms that achieve the policy targets at all Nash equilibria of the games induced by them, this paper studies a sequence of problems that are increasing in complexity in terms of the constraints they include. These problems deal with the optimization of the social welfare function (i) without any constraints, (ii) with individual constraints (constraints on the actions of individual agents), and (iii) with homogeneous (additive) joint constraints (constraints that are function of multiple agents actions). The solution of these problems combined together leads to the design of a sequence of electricity markets that achieves the objective.

\subsection{Contributions}

This study presents electricity markets that achieve the electricity policy targets (reliability, sustainability, and price efficiency) described in Section 1.1 considering the technological changes in the industry, namely demand elasticity. In addition to achieving the policy targets, the proposed electricity markets possess the following desirable properties: budget balance, individual rationality, and social welfare optimality. A market that is price efficient and possesses the above three properties is called an efficient market. These three properties are the important desired properties of electricity market in practice [24,25]; other desired properties of the electricity markets such as coalition proofness shown in [26] are beyond the scope of this research and future directions. Finally, the design provides answers to electricity policy debates described in Section 1.1. Specifically, the results show that: (1) Price efficiency is achieved without using price caps, offer caps, market monitoring or other sorts of market interventions. (2) With respect to the debate on reliability, an energy-and-capacity solution/market as well as an energy-only solution/market achieve planning reserve requirements. The energy-and-capacity solution achieves efficient subsidy but requires extra markets (capacity markets) and corresponding regulations. The energy-only solution requires more information and complex computations by the system operator, and pays more subsidy to GenCos compared to the energy-and-capacity solution. In the absence of planning reserve requirements, no capacity markets or other subsidiary mechanisms are required to achieve socially optimal investment (this is in contrast to References [11,12]). (3) With respect to the debate on carbon market vs carbon tax, the price of carbon determined by the design in the carbon permit market is the efficient taxation required to meet the carbon emission target.

\subsection{Literature Review}

In accordance with the objectives, first, a review of the literature related to market design for implementing electricity policy targets, namely, price efficiency, reliability (capacity requirements), and sustainability (carbon emission constraint) is presented. Then, a review of the literature on mechanism design that is related to the problem is presented. Finally, the results are compared with those that appear in the reviewed literature.

\subsubsection{Price Efficiency}

Uniform price auctions have been proposed for electricity markets in order to achieve price efficiency. The proposed auctions are static auctions of divisible goods. Supply function (SF), Cournot, first and second price auctions are examples of uniform price auctions proposed for electricity markets.

In practice, SF auctions are the major approach used for electricity spot markets. In SF auctions GenCos bid their cost curve and the market is cleared by dispatching the minimum cost first $[27,28]$. The same bids are sometimes used to clear multiple possible demands due to demand uncertainty or demand variation during the day [29]. In general, SF auctions have a range of equilibria and are not efficient [27]. The inefficiency of SF auctions has been studied further within the context of oligopoly models with capacity constraints [30], models with generation portfolio [31], models with a pivotal bidder [32] and network constraints [33], and duopoly models with finite piece-wise linear cost function [34]. 
Cournot auctions have been used to approximate SF electricity auctions [35]. These auctions do not achieve social welfare maximizing allocations at Nash equilibria, but they are price efficient as the price at Nash equilibria is equal to marginal cost of production [29].

First and second price auctions have been used for capacity markets [36]. While these auctions implement the socially optimal allocation at Nash equilibria for a single indivisible item, they do not maximize social welfare in problems concerned with the allocation of divisible goods. Moreover, the uniform price in these auctions is not efficient.

In addition the above uniform price auctions, Vickery-Clarke-Groves (VCG) and d'Aspremont-Gerard-Varet (AGV) have also been proposed for electricity markets [37]. VCG and AGV achieve social optimality by paying each seller an individual price that aligns their incentive with the social welfare. However, VCG and AGV auctions are not price efficient and, in addition, they can not be budget balanced and individually rational at the same time [38].

The auctions/mechanisms proposed here are price-efficient, and in addition they are budget balanced, individually rational, and social welfare maximizing. A key idea in these proposed auctions for electricity is to use a uniform price at equilibrium to achieve price efficiency and budget balance, and discriminatory prices off-equilibrium to align individuals' incentives with the social welfare. Such an idea has also been proposed during the early discussions concerning the restructuring of the British electricity markets [39].

\subsubsection{Reliability}

Implementation of reliability targets for electricity markets has been studied by considering sources of underinvestment, mainly market power and market imperfections leading to the missing money problem. Market power originates from the oligopoly nature of the market and increases in the case of inelastic demand. Market imperfections are further categorized into demand inelasticity and uncertainties. If the inelastic demand exceeds maximum capacity it results in market failure where supply and demand mismatch without any scarcity price to signal correct investment [40].

Several papers have studied underinvestment in capacity-trade competition where firms first expand their capacity and later use that capacity to compete in the trade market. These studies analyze specific forms of trade markets including Bertrand (capacity-price competition) [41], Cournot (capacity-quantity competition) [42], VCG [43], and competitive markets [44]. In the case of Bertrand and Cournot, the market equilibrium is not socially optimal. In the case of VCG and competitive markets, the outcome is socially optimal; but, VCG is not budget-balanced, and perfect competition is a strong assumption for electricity oligopolistic markets.

The methods proposed to address the issue of underinvestment and ensure meeting reliability constraints can be categorized into [44]: (i) correcting the spot (energy) markets e.g., using new market processes, lifting price caps, and price-dependent market intervention techniques [45]; (ii) using supportive energy remuneration mechanisms (ERM), e.g., operation reserve markets [46], forward generation contracts [47], and mandatory load hedging (MLH) [48]; (iii) using supportive capacity remuneration mechanisms (CRM) e.g., forward capacity obligation with capacity markets/bilateral trades (ICAP), capacity payments, strategic reserve, and reliability options $[40,48]$. Supportive mechanisms should be designed in a way that their effect automatically diminishes as the spot market imperfections are resolved [49]. These studies use a basic model of one stage investment decision followed by one stage production market.

Reliability has also been studied within the context of long-term dynamic interactions among strategic GenCos [50]. Models of long-term dynamic interactions incorporate uncertainty, symmetric, or asymmetric. For electricity industry, symmetric uncertainty has been particularly studied in the literature. Reference [51] provides an energy-only model (a model of only spot markets) for capacity expansion under uncertainty and by finding a Markov perfect equilibrium using simulations, it shows that the underinvestment problem causing high prices exists in this model. Reference [11] uses a highly stylized 
energy-only model with Cournot spot markets and provides the same results analytically. These results do not model capacity reserve requirement explicitly, but only argue that even in the absence of such requirements underinvestment exists in current markets which leads to high prices (inefficient prices). Dynamic markets with capacity reserve requirement are studied in [50]. They show the capacity market can reduce market power but provide concrete conditions for need of capacity markets. The exacerbating effect of renewables uncertainty in this context reliability is studied by [52].

This work relates to the literature of reliability as follows. Reference [53] implements reliability targets using energy-only markets but the analysis of that paper is limited to static environments and also the mechanism results in inefficient subsidy for reliability. In this paper, reliability and efficiency are achieved as follows: first the one-period problem of simultaneous decision on investment expansion and production is formulated as auctions with constraints. This study shows that in the absence of the reliability constraint, one can use the efficient spot markets to implement socially optimal investment. Then, reliability constraints are added in terms of capacity reserve requirements and characterized as homogeneous (additive) joint constraints. Implementing joint constraints in the auction design is a challenging task because it couples the GenCos' decisions, furthermore, such constraints are uneconomic and require appropriate subsidy/tax. This study proposes a method that sequentially decomposes the problem with joint constraints into two problems, an expansion decision followed by a production decision each with individual constraints only. This method implements the efficient investment and production in subgame perfect Nash equilibrium of a dynamic two-stage mechanism where at each stage an efficient auction is used. The methodology results in an efficient capacity and spot market (capacity-and-energy solution). Furthermore, this study extends the results by considering long-term interaction of strategic GenCos, which are not myopic, with symmetric uncertainty. The model of long-term interactions and uncertainty provided in this study is similar to References [11,51], but in contrast to those papers, this study takes a design approach to propose efficient markets. This study extends the decomposition technique to design a sequence of efficient capacity and spot markets.

\subsubsection{Sustainability}

Practices for charging carbon emission have been in the form of a carbon emission tax or carbon market (also carbon cap-and-trade market) [54,55]. With a fully-informed system operator, they lead to the same outcome [56]; otherwise, only an efficient carbon market can find the efficient carbon tax [57]. Moreover, calculation and continuous adjustment of the carbon tax is a difficult task [8]. However, current carbon markets are not efficient as GenCos can use their market power to pay a price for carbon that is lower than the efficient tax [9,10]. Designing efficient markets for charging carbon is an open problem [49].

This study addresses this problem by first translating the carbon constraint into a homogeneous joint constraint (constraints that are function of multiple agents' actions) via the introduction of carbon permit as auxiliary variables. Then this study uses the presented method for implementing homogeneous joint constraints, presented in the discussion of reliability, to design efficient carbon markets.

\subsubsection{Literature on Mechanism Design}

Since this study considers problems with symmetric information, the games induced by the auction/mechanisms this study proposes have symmetric information structure, therefore, this study adopts the Nash equilibrium (NE) as the solution concept for the games induced by the mechanisms for one-period problems and subgame perfect Nash equilibrium (SPNE) for games induced by mechanisms for multi-period problems.

Implementation in NE was first studied by [58] in the context of static (one-period) problems. Reference [58] determined conditions necessary and sufficient to achieve implementation of social choice functions in NE when the number $N$ of strategic users is larger than two. Furthermore, he specified a mechanism that achieves implementation 
in NE. The message space of this mechanism is, in general, infinite dimensional (as the agents report their type completely) and this is its main drawback. Additional results refining Maskin's work were presented in [59-63]. Reference [64] presented a mechanism that achieves implementation of Walrasian and Lindahl outcomes in NE when $N \geq 3$ and has a low-dimensional message space. Necessary and sufficient conditions for implementation in NE when $N=2$ were presented in $[65,66]$. A thorough discussion and survey of implementation in NE for one-period (static) problems appear in $[67,68]$.

Mechanism design has been used to solve static problems in engineering where the environment is non-Bayesian, strategic agents posses private information, and NE is the solution concept in the games induced by the mechanisms. Instances of such problems are bandwidth allocation in wired networks $[69,70]$, spectrum allocation and spectrum sharing in wired networks [71], cybersecurity and cyberinsurance [72], and energy markets [73].

Implementation in subgame perfect Nash equilibrium (SPNE) for a static problem was first studied by [74,75]; the authors determined necessary and sufficient conditions under which a social choice function is implementable in SPNE.

\subsubsection{Comparison of the Results with the Relevant Literature}

The auction/mechanisms we propose in this paper achieves the implementation of the social welfare function in subgame perfect NE, they are individually rational, budgetbalanced, and price efficient. Thus, they are efficient. Consequently, they are different from all previous work in the literature of price efficiency, reliability, and sustainability, as none of them is efficient and none of them considers all three policy targets together. In the energy-and-capacity solution, the price in the carbon market will be the efficient tax for carbon emission and the price in the capacity market will be the efficient subsidy for reliability. Furthermore, we also propose an energy-only solution by adding to the spot market demand, which can be implemented in the form of operation reserve market, and show that this solution requires more subsidy for reliability compared to the efficient energy-and-capacity solution.

The mechanisms we propose are also different from the mechanism design literature in the following way. (i) We studied the efficient auction of a divisible elastic and inelastic good. (ii) The auctions include multiple decision variables for the agents, e.g., capacity expansion and production, where one variable sets a constraint for the others (individual constraints). (iii) The auctions put constraints as a function of the decisions of multiple agents (homogeneous joint constraints). These are explained below.

The presented efficient mechanisms without constraints is inspired by price mechanism of [64] for implementing a Walrasian outcome. But this work is distinctly different from [64] because we studied designing auctions; in other words the mechanism design allocates non-strategic demand, both elastic or inelastic, among multiple strategic agents. Moreover, this study adds price efficiency as a desired feature for the design. Also, the individual and joint constraints are not present in that work.

To the best of the authors' knowledge the design of the efficient mechanism with individual and joint constraints similar to the ones appearing in this paper has been an open problem. The closest works to this work are the subgame perfect implementation of [74] and the literature following that. This study adopts similar decomposition ideas in [74] that are used for dynamic implementation of an originally static problem. But those are not price mechanisms, i.e., there is not a uniform price for agents, and moreover, the message space has large dimensionality (each agent should report his entire private information), which makes them impractical. Moreover, this study extends the results to the case where strategic non-myopic agents interact for a long horizon with symmetric uncertainty. To do this, this study takes advantage of the unidirectional constraint that some decision variables impose on the others (the capacity and carbon permission decisions set constraints for the production in the spot market, but not vice versa), to induce GenCos' myopic behavior in the spot markets at the subgame perfect Nash equilibrium of the games 
induced by the design mechanisms. This, in turn, allows us to handle the complicated long term interactions.

\section{Model}

This section first models the electricity industry. Then, it adds the policy targets. It discusses the model limitations and propose possible extensions in Section 8.

Industry Model: With some abuse of notation and for simplicity of presentation, consider a $T$-period ( $T$-year) economy, $T=\{1,2, \ldots, T, T+1\}$, consisting of $N=\{1,2, \ldots, N\}$ strategic GenCos ( $N \geq 3$ for technical issues), a non-strategic elastic demand with discount factor $\beta$ and an independent system operator (ISO). Demand elasticity is due to the new technologies including demand response management (DRM) programs and battery storage. GenCos have symmetric information. Let $e_{n, t}$ denote GenCo $n$ 's electricity production at time $t$. GenCo $n^{\prime}$ s production cost at time $t$ is $C_{n, t}^{e}\left(e_{n, t}\right)$ where $C_{n, t}^{e}\left(e_{n, t}\right)$ is strictly convex and increasing with respect to $e_{n, t}$ and $C_{n, t}^{e}(0)=0$. The demand's utility at time $t$ is denoted by $U_{t}\left(d_{t}\right)$, where $U_{t}(0)=0$ and $U_{t}\left(d_{t}\right)$ is strictly concave and increasing with respect to $d_{t}$. Note that this utility represents elastic demand. Let $\Delta x_{n, t}$ denote GenCo $n^{\prime}$ 's capacity expansion at time $t$. GenCo $n^{\prime}$ s expansion cost at time $t$ is $C_{n, t}^{x}\left(\Delta x_{n, t}\right) . C_{n, t}^{x}\left(\Delta x_{n, t}\right)$ is strictly convex and increasing with respect to $\Delta x_{n, t}$ with $C_{n, t}^{x}(0)=0$.

Initially, GenCos have capacity $x_{n, 0}, n \in N$. At the beginning of each year $t \in T$, all GenCos simultaneously and independently decide on how much to expand their existing capacities. The new capacities are built and added to the system so that the total GenCo $n^{\prime} \mathrm{s}$ capacity for generating electricity at time $t$ is $x_{n, t}=x_{n, 0}+\sum_{\tau=1,2, \ldots, t} \Delta x_{n, \tau}$. Next, demand at time $t^{+}$with utility $U_{t}\left(d_{t}\right)$ realized and is cleared in the spot market at time $t$. GenCo $n^{\prime} \mathrm{s}$ production at the spot market of time $t$ is denoted by $e_{n, t}$ and its money transfer is denoted by $r_{n, t}$. The timeline of events during year $t$ is shown in Figure 1 . Note that this model has only considered one spot market between expansion decisions just for simplicity of the presentation and the results hold even if there are numerous spot markets in each year between expansion decisions (which is the real case). Alternatively, this single market can also be interpreted as the aggregate of all spot markets in between expansion decisions. In the same way the assumption that the new capacity is available in the same year is for simplicity of presentation, and the results hold with multi-stage delay in expansions (in reality it takes multiple years to build new power plants). The model can be updated to include both of these, and the results will not change, but the presentation will be more complicated (see Section 8).

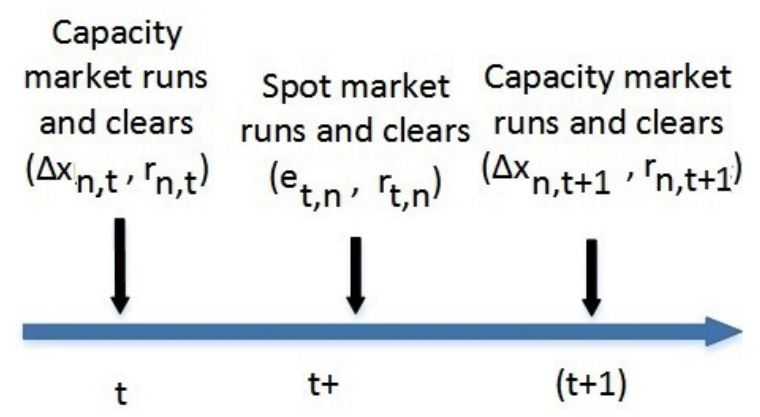

Figure 1. Timeline of events in the electricity industry during period $t$ to $t+1$.

After the final year, at time $T+1$, each GenCo $n \in N$ sells its entire capacity with price $\eta$, gains a value of $V_{c}\left(x_{n, T+1}\right)=\eta \times x_{n, T+1}$, and leaves the economy.

Next, this study models the ISO. The ISO is a social welfare that maximizes, designs the mechanisms, and runs the auctions, but does not participate in those.

Next, the model's information structure is presented. This model assumes that GenCos have symmetric information, thus they know each others' type (cost functions and initial capacities), and any time $t$ they know all the information history up to $t$. The information history at any time $t$ includes all the expansion decisions, bids in the market, 
market outcomes for all GenCos, and demand values (assume the ISO announces the market outcomes and demand in case they are not already available to the GenCos). Since information is symmetric, this study adopts the Nash equilibrium for one-period problems and subgame perfect Nash equilibrium for multi-period problems as solution concepts for the mechanism design. Note that the ISO does not necessarily know the type of the GenCos (their cost functions $C_{n, t}^{e}(),. C_{n, t}^{x}(),. n \in N, t \in T$, and initial capacity $x_{n, 0}, n=0,1,2, \ldots$ ), but knows the spaces where $C_{n, t}^{e}, C_{n, t}^{x}$, and $X_{n, 0}$ belong to.

This completes the description of the model for the industry. Note that while this study has not considered network constraints, the above model allows isolating and capturing some of the main dynamics of the electricity industry needed to study and understand the policy issues proposed in Section 1.1 (oligopolistic market with certain industry constraints). This study discusses in Section 8 about other important features of electricity industry and how this work can be expanded to capture them.

Policy Targets Model: This study models the policy targets i.e., reliability, sustainability, and price efficiency. The reliability target is in the form of the following planning reserve requirements:

$$
\sum_{n \in N} x_{n, t} \geq \underline{x}_{t}, \quad \forall t \in T
$$

This means that at each time $t$, there is a minimum capacity requirements denoted by $\underline{x}_{t} \quad \underline{x}_{t}$ is calculated by the system operator based on future demand prediction scenarios, uncertainties in the supply side e.g., due to renewable, and degree of reliability required by the ISO/TSO (e.g., one in ten year black out or alternative requirements) [76].

This study models the system operator's sustainability target as follows. Consider $\alpha_{n, t}$ to be the carbon factor for the technology of producer $n$ used for producing electricity at time $t$. Then at time $t$, the sustainability target restricts carbon emission by carbon cap $\bar{b}_{t}$ is described by

$$
\sum_{n \in N} \alpha_{n, t} e_{n, t} \leq \bar{b}_{t}
$$

Price efficiency requires that a uniform price is paid to all GenCos and this price is equal to the marginal cost of production for those producers whose production is positive and is not saturated by their capacity. Assuming $p_{n, t}$ to be the price paid to GenCo $n$ at time $t$, then

$$
p_{n, t}=C_{n, t}^{\prime}\left(e_{n, t}\right) \quad \text { if } \quad 0<e_{n, t}<x_{n, t}, \quad \text { and } \quad p_{n, t}=p_{t} \quad \forall n \in N, t \in T .
$$

Next, a methodology is described for implementing the above policy targets for the proposed industry model. Specifically: (i) this study studies a sequence of auctions that are increasing in the complexity of the constraints they include (Sections 3-5); (ii) this study combines the solutions to these auctions to achieve its objective (Section 6).

\section{Efficient Auctions of a Divisible Good}

In this section, a design of a static (one-time period) efficient auction for allocating a divisible good without any constraints is presented. This problem serves as the fundamental building block of the development in the following sections where this study considers one-time auctions with individual or homogeneous (additive) joint constraints. The problem is also a fundamental building block of the approach to multi-period auctions. Inspired by [64], the design uses discriminatory/individual prices to implement social welfare maximizing outcome. However, these individual prices become the same at equilibrium to ensure price efficiency. A discussion on the interpretation of the design is provided in Section 3.3.

We consider two separate cases of price elastic and price inelastic divisible goods. The proposed efficient auctions with elastic divisible good are used to design spot markets with 
elastic demand. The proposed efficient auction of inelastic divisible good are used in the next sections to design capacity market, and carbon permit market.

\subsection{Model: Electricity Spot Markets}

Based on the model of Section 2, this study considers a single spot market without constraints. Since this study studies only a one-period market, for ease of presentation, the time index is dropped in the model of this section. In Appendix B, this study extends the results to the case where both long-term interactions among agents and symmetric uncertainty exist. Long-term analysis of the electricity system with uncertainties is studied with different techniques in [77-79]. We study auctions with both elastic and inelastic divisible goods here. We use elastic demand to model the spot market. This is due to new technologies including demand response management (DRM) and battery storage that improve the elasticity of demand.

The social-welfare maximizing allocation for elastic demand is given by the solution of the following problem.

$$
\max _{e_{n}, n \in N} U\left(\sum_{n \in N} e_{n}\right)-\sum_{n \in N} C_{n}^{e}\left(e_{n}\right)
$$

If the demand is inelastic and equal to $\underline{d}$ then, the social welfare maximizing problem is

$$
\max _{e_{n}, n \in N}-\sum_{n \in N} C_{n}^{e}\left(e_{n}\right) \quad \text { s.t. } \quad \sum_{n \in N} e_{n} \geq \underline{d}
$$

Note that both of the above problems are strictly convex optimizations with a convex non-empty domain and therefore, have a unique solution.

\subsection{Design/Mechanism}

We present two auction mechanisms, one for elastic and one for inelastic demand. The two mechanisms are only different in their payment methods.

A game form/mechanism is described by $(\mathcal{M}, g)$, where $\mathcal{M}$ is the message/strategy space and $g: \mathcal{M} \rightarrow \mathcal{A}$ is the outcome function, a function from the message space $\mathcal{M}$ to the space $\mathcal{A}$ of allocations. Consider the following mechanism.

Message space Choose GenCo $n$ 's message space, $\mathcal{M}_{n}$, its message, $m_{n}$, and the message space of the mechanism, $\mathcal{M}$, to be

$$
\mathcal{M}_{n}:=\mathbb{R} \times \mathbb{R}_{+}, n \in N, \quad m_{n}=\left(\hat{e}_{n}, \hat{p}_{n}\right), \quad \mathcal{M}:=\left(\mathcal{M}_{1} \otimes \mathcal{M}_{2} \otimes \ldots \otimes \mathcal{M}_{N}\right),
$$

respectively, where $\hat{e}_{n}$ denotes the amount of electricity GenCo $n$ proposes to produce, and $\hat{p}_{n}$ denotes the price GenCo $n$ proposes to charge per unit of electricity; $\hat{p}_{n}$ is restricted by $\hat{p}_{n} \geq 0$.

Allocation Space The allocation space of the mechanism, $\mathcal{A}$, GenCo $n^{\prime}$ s allocation space, $\mathcal{A}_{n}$, and GenCo $n$ 's allocation $a_{n}$ are

$$
\mathcal{A}:=\left(\mathcal{A}_{1} \otimes \mathcal{A}_{2} \otimes \ldots \otimes \mathcal{A}_{N}\right), \quad \mathcal{A}_{n}:=\left[0, x_{n}\right] \times \mathbb{R}, n \in N, \quad a_{n}=\left(e_{n}, r_{n}\right),
$$

respectively, where $e_{n}$ is the amount GenCo $n$ is scheduled to produce, and $r_{n}$ is the payment to GenCo $n$.

Outcome function Define $g: \mathcal{M} \rightarrow \mathcal{A}$ as follows. For each $m:=\left(m_{1}, m_{2}, \ldots, m_{N}\right) \in \mathcal{M}$ set $g(m)=(e, r)=\left(e_{1}, \ldots, e_{N}, r_{1}, \ldots, r_{N}\right)$ where

$$
e_{n}=\hat{e}_{n} .
$$

For elastic demand the payments are

$$
r_{n}^{\text {elas. }}=\hat{p}_{n+1} \hat{e}_{n}-\hat{p}_{n}^{-0.5}{\zeta_{n}^{\text {elas. }}}^{2},
$$




$$
\zeta_{n}^{\text {elas. }}=D\left(\hat{p}_{n+1}\right)-\sum_{n \in N} \hat{e}_{n}, \quad D(\hat{p})=\left(U^{\prime}\right)^{-1}(\hat{p}), \quad \hat{p}_{N+1}:=\hat{p}_{1} .
$$

For inelastic demand the payments are

$$
\begin{gathered}
r_{n}^{\text {inelas. }}=\hat{p}_{n+1} \hat{e}_{n}-\left(\hat{p}_{n}-\hat{p}_{n+1}\right)^{2}-2 \hat{p}_{n} \zeta^{\text {inelas. }^{2}}, \\
\zeta^{\text {inelas. }}=\left(\underline{d}-\sum_{n \in N} \hat{e}_{n}\right)^{+}, \quad p_{N+1}:=p_{1} .
\end{gathered}
$$

The indexing used for allocations above is for simplicity of presentation; there are other methods for allocation that induce the same results without any indexing. Refer to one such method at the end of Section 3.3.

\subsection{Interpretation of the Design}

The key challenges in the design of an efficient mechanism for the problem considered in this section are (1) dealing with the strategic GenCos' market power; (2) incentivizing the strategic GenCos to collectively meet the price-taking demand.

Since the mechanism designer, i.e., the ISO, cannot alter the producers' cost functions, $C_{n}^{e}(),. n=1,2, \ldots, N$, even if they knew their functional form, the only way they could achieve their objective (social welfare maximization) is through the use of appropriate tax incentives/tax functions. For the case of elastic demand, the tax function is $r_{n}^{\text {elas. }}=$ $\left(\hat{p}_{n+1} \hat{e}_{n}\right)+\left(-\hat{p}_{n}^{-0.5}\right.$ zelas. $\left._{n}^{2}\right)$. For the case of inelastic demand, it is $r_{n}^{\text {inelas. }}=\left(\hat{p}_{n+1, t} e_{n}\right)+$ $\left(-\left(\hat{p}_{n}-\hat{p}_{n+1}\right)^{2}-2 \hat{p}_{n} \zeta^{\text {inelas. }}{ }_{t}^{2}\right)$.

Allowing discriminatory individual prices charged to GenCos (off-equilibrium) provides the flexibility required for implementing social welfare maximizing generation by eliminating market power. This is achieved by offering each producer a price per unit of its production that does not depend on its own price proposal, that is, it is not under its own control. Such an offer induces price-taking behavior among the producers. One way to achieve price-taking behavior is to arbitrarily index the GenCos between 1 and $N$, and then pay GenCo $n$ according to the price proposal $p_{n+1}$ of GenCo $n+1$, with the convention that $p_{N+1}=p_{1}$. The indexing is arbitrary but known to all GenCos. The term $r_{n, 1}=\left[\hat{p}_{n+1, t} e_{n}\right]$ specifies the amount GenCo $n$ receives for its production $e_{n}$.

Incentivizing the producers to bid the same prices can be achieved by a penalty term for each producer $n$ that depends on his own price. In a similar manner, incentivizing the strategic producers to collectively meet the demand is achieved by penalizing overproduction or under-production. For inelastic demand, the incentive provided to all producers to bid the same price per unit of capacity is described by the term $-\left(\hat{p}_{n, t}-\right.$ $\left.\hat{p}_{n+1, t}\right)^{2}$, the incentive provided to all producers to collectively propose a total production that is at least equal to the demand, $\underline{d}$, is captured by the term $-2 \hat{p}_{n, t} \zeta^{\text {inelas. }}{ }_{t}$, which is a penalty for underproduction. For the elastic demand, the term $\hat{p}_{n}^{-0.5} \zeta_{n}^{\text {elas. }}{ }^{2}$ ensures that the equilibrium price is equal to the marginal utility of the demand at the total amount of production. Under this price, all the electricity supplied at the suggested price is bought and used by the demand. Note that $D(p)$ is the optimal demand for price $p$, because it is the solution of the optimization problem below:

$$
D(p) \in \arg \max _{d \geq 0} U(d)-p d .
$$

Since the above optimization is strictly concave, it has a unique solution.

The above incentives represent tax/subsidy payments the ISO collects from/provides to the strategic GenCos, which, in addition to achieving the above stated objectives, allow the ISO to align the GenCos productions with the social welfare maximizing production profile. 
We note that, according to the above guidelines, different penalty terms can be designed for efficient auctions and those terms proposed here are just one possible design. An alternative design that does not require indexing of the GenCos is to pay every GenCo the average of the prices proposed by the other GenCos. This alternative design process, at equilibrium, has the same properties as the design proposed in Section 3.2.

\subsection{Comparison of the Elastic and Inelastic Static Auctions}

We now contrast the two auction mechanisms proposed for elastic and inelastic divisible good. The key differences appear in the specification of the tax function that provides incentives to strategic GenCos to align their interests/goals with those of the ISO. In the case of elastic demand, the money the demand pays defines the amount of electricity it consumes. Thus, the price per unit of power should be less than or equal to the marginal utility of consumption at the amount injected to the market. This requirement expresses the budget balance property the mechanism should have. In the case of inelastic demand, budget balance is not required because the demand pays to the GenCos any amount it is charged. In return, GenCos' production profile $e=\left(e_{1}, e_{2}, \ldots, e_{N}\right)$ must meet the demand, that is it must satisfy an inequality constraint of the form $\sum_{n=1, \ldots, N} e_{n} \geq \underline{d}$. The difficulty in this case is designing a price efficient mechanism (a mechanism where the price is limited by marginal cost of production), which also satisfies $\sum_{n=1, \ldots, N} e_{n} \geq \underline{d}$.

As a result of these differences, the two mechanisms are different in the way GenCos contribute to the tax payment that is imposed so as to achieve an efficient allocation at equilibrium.

\subsection{Properties of the Proposed Mechanisms}

The properties possessed by the two proposed mechanisms are presented in this section. Consider Nash equilibrium (NE) as the solution concept. Denote the equilibrium

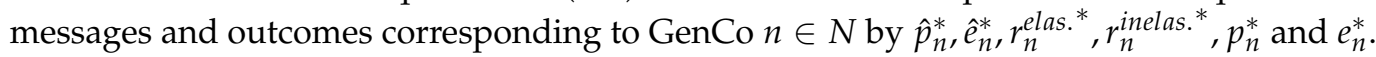

First define the notion of a trivial equilibrium (Definition 1); it is shown that the auction for inelastic demand has exactly one trivial NE (Lemma 2). Hereafter, for simplicity of presentation whenever NE is referred to, it is meant non-trivial NE unless otherwise stated. It is proven that at any equilibrium (trivial or non-trivial) outcome of the game induced by the above mechanisms, supply and demand meet each other (Lemma 1). Use Lemmas 1 and 2 to prove price efficiency, budget balanced, social welfare maximization, and individual rationality at any Nash equilibrium of the game induced by the mechanisms in Theorems 1-4, respectively. The proofs of all these properties are presented in Appendix A. This section is concluded by discussing uniqueness of the NE, Pareto dominance of the non-trivial NE compared to the trivial NE for the inelastic demand, and the anonymity of the mechanisms.

Definition 1 (TRIVIAL EQUILIBRIUM). An equilibrium where price bids, $\hat{p}_{n}^{*}$, are zero for all $n \in N$ is defined to be a trivial equilibrium.

Lemma 1 (MARKET CLEARANCE). At every non-trivial equilibrium, supply meets demand, i.e., for elastic demand and inelastic demand, for all $n \in \mathcal{N}$,

$$
\begin{array}{r}
\zeta_{n}^{\text {elas. }}{ }^{*}=0, \quad \text { or equivalently } D\left(\hat{p}_{n+1}^{*}\right)-\sum_{n \in N} \hat{e}_{n}^{*}=0, \\
\zeta^{\text {inelas. }}{ }^{*}=0, \quad \text { or equivalently } \quad\left(\underline{d}-\sum_{n \in N} e_{n}^{*}\right) \geq 0 .
\end{array}
$$

Theorem 1 (PRICE EFFICIENCY). The auctions proposed for elastic and inelastic demand are price efficient at any non-trivial equilibrium $m^{*}$ of the games induced by the mechanisms; that is, 
there is a uniform price equal to the marginal cost of the next one unit of production by producers, i.e., for every producer $n \in N$, which is not saturated,

$$
\begin{aligned}
\hat{p}_{n}^{*}=\hat{p}_{n+1}^{*} & =\hat{p}^{*}, \\
r_{n}^{*} & =\hat{p}^{*} \hat{e}_{n}^{*} \\
\left.\frac{\partial r_{n}}{\partial \hat{e}_{n}}\right|_{m^{*}} & =\hat{p}^{*}, \\
\hat{p}^{*} & =C^{\prime}\left(\hat{e}_{n}^{*}\right) .
\end{aligned}
$$

Theorem 1 shows that at equilibrium all non-saturated producers propose the same price per unit of electricity (Equation (16)). This equilibrium price is both the average price (Equation (17)) and the marginal price (Equation (18)), and is efficient in the sense that it is equal to the marginal cost of production for non-saturated producers (Equation (19)). This theorem shows that the proposed mechanisms incentivize producers to reveal at equilibrium their true cost of producing the next unit of electricity provided that they still have free capacity. This marginal cost is the same for all producers with free capacity, and therefore, would be the marginal cost of a hypothetical entity who would own all the producers.

Theorem 2 (BUDGET BALANCED). The proposed auctions are budget balanced at any trivial or non-trivial equilibrium of the game induced by them. For elastic demand, if $\sum_{n \in N} \hat{e}_{n}^{*}>0$, this means that the equilibrium price is equal to marginal utility of the demand,

$$
\hat{p}^{*}=U^{\prime}\left(\sum_{n \in N} \hat{e}_{n}^{*}\right) .
$$

Note that, in contrast to the auction for elastic demand, budget balance is always achieved in the auction for inelastic demand by charging demand the total payment to the GenCos.

Theorem 3 (NASH IMPLEMENTATION/SOCIAL OPTIMALITY). At any non-trivial equilibrium of the game induced by the proposed auction for elastic or inelastic demand, the allocations of the demand to GenCos are social welfare maximizing, i.e., they are equal to the unique solutions of the Problems (4) and (5), respectively.

Theorem 1 determines the price bids and Theorem 3 determines the production bids at equilibrium. Using these bids, one can establish individual rationality.

Theorem 4 (INDIVIDUAL RATIONALITY). The proposed auction mechanisms for elastic and inelastic demand are individually rational, that is at any trivial or non-trivial NE of the games induced by the mechanisms and for every GenCo $n \in N$, the corresponding allocations $\left(e_{n}^{*}, r_{n}^{\text {elas. }}{ }^{*}\right)$ and $\left(e_{n}^{*}, r_{n}^{\text {inelas. }}{ }^{*}\right)$ are weakly preferred to the allocation $(0,0)$ (the allocation a strategic producer receives when they do not participate in the market).

Theorem 4 shows that each strategic producer voluntarily participates in the electricity market.

Lemma 2 (UNIQUENESS OF TRIVIAL NE). The game induced by the proposed auction for inelastic demand has, in addition to the unique non-trivial $N E$, exactly one trivial $N E$, where for all $n \in N, p_{n}^{*}=0$ and $e_{n}^{*}=0$. The game induced by the auction for elastic demand does not have any trivial equilibrium if in the solution to Problem (4), $e_{n}^{*}$ is not zero for all $n \in N$, otherwise it has a unique trivial equilibrium where $p_{n}^{*}=0$ and $e_{n}^{*}=0$ for all $n \in N$.

Remark 1 (UNIQUENESS OF NONTRIVIAL NE). The games induced by the proposed mechanisms have a unique non-trivial NE. This is because by Theorem 3 , the bids for electricity production 
at equilibrium are equal to the unique solution of Problem (4) for elastic demand and Problem (5) for inelastic demand. Furthermore, by Theorem 1, the price bids of all the GenCos is the same at equilibrium, and equal to marginal cost of producing the next unit of electricity.

Remark 2 (PARETO DOMINANCE). Since $(0,0)$ is the outcome of the trivial NE of the game induced by the mechanism, Theorem 4 shows that the unique non-trivial NE Pareto dominates the trivial NE.

Remark 3 (ANONYMITY). The proposed mechanisms are anonymous at equilibrium. In other words, at equilibrium payments and productions are independent of the GenCos' indexing. To see this, note that at equilibrium the electricity produced by each GenCo is according to the social welfare maximizing production which is independent of the GenCos' indexing. Furthermore, as shown in Lemma 1 (Equation (16)) the price paid per unit of production at equilibrium is the same for all GenCos and independent of the GenCos' indexing, and the penalty terms in the tax function are equal to zero. Note that in alternative designs presented at the end of Section 3.3, the indexing is relaxed, which induces anonymity.

\section{Efficient Auctions with Individual Constraints}

In this section, we study one-time period auctions where sellers have individual constraints. This study considers a dynamic two stage setup within a one-period framework where the agents (i.e., GenCos) decide about their investment in the first stage and sell their product in the second stage. The constraints in the second stage are assumed to be individual and due to agents' individual investments. This study shows that with individual constraints, use of the auctions proposed in Section 3.2 in the second stage results in efficiency without any need for additional prices, corresponding markets for discovering these prices in the first stage, or other instruments.

We study these auctions within the context of GenCo's capacity expansion without reliability constraints. GenCos decide their capacity expansion in stage one and then sell electricity to demand in stage two. In this section, Section 5, and Appendix B, this study focuses on the case of elastic demand; the extension of techniques and results to corresponding problems with inelastic demand can be achieved in a similar way. This study designs a capacity-trade mechanism (Section 4.2), and show that it is efficient (Section 4.3, Theorem 5). Moreover, this study proves that the GenCos use all of the expanded capacity in the spot market and the spot market price covers their investment cost (Theorem 6).

In Section 4.4, the results are interpreted. Furthermore, in Section 6, results are used to compare the proposed efficient electricity markets with an energy-only solution to reliability.

\subsection{Model: Investment without Reliability Constraint}

Consider the model of Section 2 restricted to the following capacity-trade competition: GenCos individually decide on their investment at time 1 (stage 1) and then the demand is auctioned at the spot market of time $1^{+}$(stage 2). GenCos' production is limited by their individual capacity and their capacity expansion is limited by their individual expansion limits. The social-welfare maximizing allocation for elastic demand is a solution to the problem

$$
\begin{array}{rl}
\max _{e_{n}, \Delta x_{n}, n \in N} & U\left(\sum_{n \in N} e_{n}\right)-\sum_{n \in N} C_{n}^{e}\left(e_{n}\right)-\sum_{n \in N} C_{n}^{x}\left(\Delta x_{n}\right) \\
\text { s.t. } & 0 \leq e_{n} \leq x_{n, 0}+\Delta x_{n} \quad \forall n \in N \\
& 0 \leq \Delta x_{n} \leq \overline{\Delta x}_{n} \quad \forall n \in N
\end{array}
$$

Problem (21) is a strictly convex optimization problem with a convex non-empty domain; therefore, it has a unique solution. 


\subsection{Design/Mechanism}

This study proposes the following dynamic mechanism for capacity-trade competition. GenCos simultaneously decide on their expansion $\Delta x_{n}$ at time 1 (Stage 1 ). Then at time $1^{+}$(Stage 2), they participate in the efficient auction (spot market) for elastic demand proposed in Section 3.2. Note that due to constraint in Equation (22), GenCo $n$ 's message space in the spot market is limited by $0 \leq \hat{e}_{n} \leq x_{n}=x_{n, 0}+\Delta x_{n}$.

\subsection{Properties of the Proposed Mechanism}

This study considers subgame perfect Nash equilibrium (SPNE) as the solution concept for the two-stage problem that arises within one-period time, and study the equilibrium properties of the proposed capacity-trade mechanism. Denote the equilibrium outcome of the game induced by the mechanism by $\Delta x_{n}^{*}, e_{n}^{*}$ and $p_{n}^{*}$. The properties of the mechanism are summarized by Theorems 5 and 6 . The proofs of Theorems 5 and 6 are special cases of the proofs of Theorems 7 and 8, respectively, in Section 6.

Theorem 5 (EFFICIENCY). The game induced by the proposed mechanism for capacity-trade competition (capacity expansion and production) has a unique subgame perfect Nash equilibrium (SPNE). At this unique SPNE, the proposed mechanism has the following properties: (i) implements socially efficient investment and production corresponding to Problem (21), (ii) is individually rational, (iii) results in efficient price in the spot market, and (iv) is budget balanced.

GenCos cover their cost of investment when their capacity becomes saturated and therefore, the market price is above their marginal cost of production. The following Theorem shows that the GenCos' investment on capacity is always covered, and it is covered at its marginal cost.

Theorem 6 (SATURATION \& SCARCITY PRICE). If GenCo n's expansion at time 1 is positive, i.e., $\Delta x_{n}^{*}>0$, then its production in the spot market at time $1^{+}$is saturated i.e., $e_{n}^{*}=x_{n, 0}+\Delta x_{n}^{*}$. Furthermore, the equilibrium price in the spot market covers the investment at marginal cost, that is,

$$
p^{*}=C_{n}^{e^{\prime}}\left(e_{n}^{*}\right)+C_{n}^{x \prime}\left(\Delta x_{n}^{*}\right)
$$

\subsection{Interpretation of the Design}

The one-period mechanism of Section 4.2 consists of two stages. In the first stage the GenCos decide for their investments independently and without any coordination mechanism such as an auction. In the second stage they participate in an auction for selling electricity. The second stage is similar to the one-stage mechanism of Section 3.2. The results of Section 3.5 ensure price efficiency, social welfare-maximization, budget balance, and individual rationality for any amount of investment that the GenCos make in the first stage. It is worth noting that the second stage of the mechanism generates the right (correct) signals for investment in the first stage. As a consequence, at equilibrium, the amount of investment the GenCos make is social welfare maximizing and is determined by the solution of problem (21). Note that such an efficient investment is implemented without any coordinator/coordinating mechanism in the first stage; this happens because each GenCo's decision in the first stage sets constraints only on his own production level in the second stage (i.e., individual constraints).

\section{Efficient Auctions with Homogeneous (Additive) Joint Constraints}

Joint constraints are public (uneconomic) constraints and their implementation requires appropriate tax/subsidy, which is in contrast to the implementation of the individual constraints of Section 4. To this end, a decomposition technique is introduced for design and analysis of the auctions. The one-time period optimization problem with homogeneous joint constraints is decomposed into a sequence of optimizations with individual constraints, and then implement them by a sequence of efficient auctions with individual 
constraints. The equilibrium price in the market corresponding to each homogeneous joint constraint determines the efficient tax/subsidy needed to implement that constraint. This study shows that the auctions proposed are efficient (Theorem 7) and discovers the efficient tax/subsidy for implementation of the homogeneous joint constraint (Theorem 8). In Appendix B, the results for one-time period auction of this section are extended to long-horizon interaction of agents.

This study presents a decomposition technique and results on homogeneous joint constraints through the study of reliability constraints (planning reserve requirements) for electricity investment where in the first stage the GenCos decide their expansion and in stage two they sell electricity in the spot market. In contrast to the setup of Section 4, here there is minimum expansion that GenCos should meet collectively due to reliability constraints. Later, Section 6 discusses how to implement carbon emission constraints using the results on auctions with homogeneous joint constraints.

\subsection{Model: Electricity Investment with Reliability Constraint}

This study uses the one-time period model of Section 2 and add the reliability constraint to the model of Section 4.1. The social welfare maximizing investment and production are a solution to the Problem (25)

$$
\begin{array}{rl}
\max _{e_{n}, \Delta x_{n}, n \in N} & U\left(\sum_{n \in N} e_{n}\right)-\sum_{n \in N} C_{n}^{e}\left(e_{n}\right)-\sum_{n \in N} C_{n}^{x}\left(\Delta x_{n}\right) \\
\text { s.t. } \quad & 0 \leq e_{n} \leq x_{n, 0}+\Delta x_{n} \quad \forall n \in N \\
& \sum_{n \in N}\left(x_{n, 0}+\Delta x_{n}\right) \geq \underline{x}
\end{array}
$$

Problem (25) is a strictly convex optimization with a convex non-empty domain; therefore, it has a unique solution.

\subsection{Design/Mechanism}

This study decomposes Problem (25) and implement its solution with separate markets. The decomposition and implementation method presented here is for reliability constraints but the method can be used for problems with other convex homogeneous joint constraints.

Decomposition: Consider vector $X=\left[x_{1}, x_{2}, \ldots, x_{N}\right]$ where $x_{n}=x_{n, 0}+\Delta x_{n}$. Problem (25) is equivalent to the following two sequential optimization problems.

$$
\begin{array}{cl}
\max _{\Delta x_{n}, n \in N} & V^{+}(X)-\sum_{n \in N} C_{n}^{x}\left(\Delta x_{n}\right) \\
\text { s.t. } & 0 \leq \Delta x_{n} \quad \forall n \in N \\
& \sum_{n \in N} x_{n} \geq \underline{x},
\end{array}
$$

followed by

$$
\begin{array}{r}
V^{+}(X)=\max _{e_{n}, n \in N} U\left(\sum_{n \in N} e_{n}\right)-\sum_{n \in N} C_{n}^{e}\left(e_{n}\right) \\
\text { s.t. } 0 \leq e_{n} \leq x_{n} \quad \forall n \in N .
\end{array}
$$

Market Design: Inspired by the above decomposition, this study proposes the following dynamic market design. First at time 1, the amount of capacity required for reliability, $\underline{x}$, is auctioned among the GenCos using efficient inelastic capacity market. The design of this market is the same as the efficient inelastic spot market proposed in Section 3. Next, at time $1^{+}$, the elastic demand $U(d)$ is auctioned among the GenCos using an efficient elastic spot market that is the same as that of Section 3. Note that due to constraint in Equation (32), the GenCo $n^{\prime}$ 's message space in the spot market is limited by $0 \leq \hat{e}_{n} \leq x_{n}$, 
furthermore, due to the constraint in Equation (29), its expansion proposals in the capacity market should be non-negative.

Proof of the properties of this dynamic market are in the next section.

\subsection{Properties of the Proposed Mechanism}

The properties of the proposed dynamic auction for implementing reliability constraints are summarized by Theorems 7 and 8 . Appendix B extends these properties to multi-period problems with symmetric uncertainty, and the proof of Theorems 7 and 8 is provided for the multi-period problems with symmetric uncertainty.

Theorem 7 (EFFICIENCY). The mechanism proposed for auctions with homogeneous joint constraints is individually rational, budget balanced, and price efficient both in the capacity market and in the spot market. Furthermore, if the solution of Problem (25) results in non-negative total expansion i.e., $\sum_{n \in N} \Delta x_{n}^{*}>0$, then the game induced by the proposed mechanism has a unique non-trivial SPNE, and the allocations corresponding to this SPNE are the same as the solution to Problem (25). The game induced by the proposed mechanism has also a trivial SPNE where the investments on capacity expansion $\Delta x_{n}, n \in N$, are equal to zero. The non-trivial SPNE Pareto dominates the trivial SPNE.

In contrast to auctions with individual constraints, the results of Theorem 6 on saturation and scarcity price results of Theorem 6 do not necessarily hold for the auctions with homogeneous joint constraints. In other words, it is possible that a GenCo expands its capacity without using the expanded capacity in the spot market. However, the price in the capacity market is efficient (Theorem 8).

Theorem 8 (EFFICIENT SUBSIDY/TAX). At the non-trivial SPNE of the game induced by the mechanism of Section 5.2, the equilibrium price $p^{c *}$ in the capacity market, is equal to the efficient level of subsidy/tax per unit of capacity expansion needed to implement the joint constraint in Equation (27), that is

$$
p^{c *}=C_{n}^{x^{\prime}}\left(\Delta x_{n}\right)+C_{n}^{e^{\prime}}\left(e_{n}\right)-p^{*}
$$

where $p^{*}$ is the equilibrium price in the spot market. This equilibrium price in the capacity market goes to zero if the joint constraint is not binding.

\subsection{Interpretation of the Mechanism}

The one-period mechanism of Section 5.2 consists of two stages. At each stage, a central auction is used to coordinate the GenCos' decisions/incentives. But these two stages have different interpretations. In the second stage the GenCos participate in an auction for selling electricity. The second stage auction is similar to the one-stage mechanism of Section 3.2. Therefore, the results of Section 3.5 ensure price efficiency, social welfaremaximization, budget balance, and individual rationality for any amount of investment that the GenCos make in the first stage. However, in contrast to the results in Section 4, the efficient auction in the second stage alone is not sufficient to send back appropriate signals to GenCos for efficient investment in the first stage. This is due to the fact that each GenCo's decision in the first stage is subject to a joint constraints for all GenCos. In other words, every GenCo's investment decision has an impact on the other GenCos' decisions (by the reliability constraint that they should jointly meet). Therefore, in the first stage, there is need for a coordinating mechanism that can complement the investment signals from the second stage auction and align the GenCos' incentives toward efficient investment. This coordinating mechanism is the auction used in the first stage. It is worth noting that when the joint constraints on the GenCos are homogeneous according to the decomposition technique of Section 5.2, the efficient auction of Section 3.2 can be used for the first stage. This is not true when the joint constraints on the GenCos are non-homogeneous. 


\section{Electricity Markets for Achieving Policy Targets}

This study uses the results of Sections 3-5 along with their extensions in Appendix B to design reliable, sustainable, and price efficient electricity markets. Also, discussion of how results contribute to the electricity policy debates of Section 1.1 are provided.

\subsection{Model: Sustainable, Reliable, and Price Efficient Electricity}

Considering reliability, sustainability, and price efficiency as desired features of the market, this study poses the question of the social welfare maximizing investment and production plans. These plans are functions of the information history. At year $t$, denote the information history for expansion by $h_{t}$, the information history for production by $h_{t^{+}}$, and their spaces by $H_{t}, H_{t^{+}}$, respectively. Define $E_{1: t}\left(x_{0}, \Delta x_{1: t}\right)$ to be the space of all possible electricity productions based on the initial capacity $x_{0}$ and capacity expansions $\Delta X_{t}$. Then,

$$
\begin{aligned}
h_{t} & :=\left\{x_{1: N, 0}, e_{1: N, 1: t-1}, \Delta x_{1: N, 1: t-1}\right\} \\
& \in H_{t}:=\left(X_{1: N, 0}, E_{1: t-1}\left(X_{1: N, 0}, \Delta X_{1: N, 1: t-1}\right), \Delta X_{1: N, 1: t-1}\right) \\
h_{t^{+}} & :=\left\{x_{1: N, 0}, e_{1: N, 1: t-1}, \Delta x_{1: N, 1: t}\right\} \\
& \left.\in H_{t^{+}}:=\left(X_{1: N, 0}, E_{1: N, 1: t-1}\left(X_{1: N, 0}, \Delta X_{1: N, 1: t-1}\right)\right), \Delta X_{1: N, 1: t}\right)
\end{aligned}
$$

GenCo $n$ 's investment plan/strategy, $n \in N$, at year $t$ is denoted by $\sigma_{n, t}: H_{t} \rightarrow$ $\left[0, \overline{\Delta x}_{n, t}\right]$ and its production plan/strategy at year $t$ is denoted by $\gamma_{n, t}: H_{t^{+}} \rightarrow\left[0, x_{n, t}\right]$. Denote the space of all such strategies by $\Sigma_{n, t}$ and $\Gamma_{n, t}, t=1,2, \ldots, T$, respectively, and define

$$
\gamma:=\left\{\gamma_{n, t}: n \in N, t \in T\right\}, \quad \sigma:=\left\{\sigma_{n, t}: n \in N, t \in T\right\} .
$$

The investment and production strategies that maximize the social welfare are the solution of the following problem:

$$
\begin{array}{ll} 
& \max _{\sigma_{n, t} \in \Sigma_{n, t}, \gamma_{n, t} \in \Gamma_{n, t}: n \in N, t \in T}\left[\sum_{t \in T} \beta^{t} U_{t}\left(\sum_{n \in N} e_{n, t}\right)-\right. \\
& \left.\sum_{t \in T} \sum_{n \in N} \beta^{t}\left\{C_{n, t}^{x}\left(\Delta x_{n, t}\right)+C_{n, t}^{e}\left(e_{n, t}\right)\right\}+\beta^{T+1} \sum_{n \in N} \eta x_{n, T}\right] \\
\text { s.t. } \quad & \forall t \in T, n \in N, \forall u, v \in V \\
& 0 \leq e_{n, t} \leq x_{n, 0}+\sum_{\tau=1, \ldots, t} \Delta x_{n, \tau} \\
& 0 \leq \Delta x_{n, t} \\
& \sum_{n \in N} x_{n, t} \geq \underline{x_{t}} \\
& \sum_{n \in N} \alpha_{n, t} e_{n, t} \leq \bar{b}_{t}
\end{array}
$$

Note that (41) is the capacity constraint for reliability and (42) is the cap on the total carbon emission. The carbon constraint is non-homogeneous because of different carbon emission factors $\alpha_{n, t}$ among GenCos.

Within the context of the setup above, budget balance and individual rationality are discussed. Budget balance is defined when the demand is elastic and requires the sum of money paid to the producers to be equal to the money collected from the demand. The demand is non-strategic (price-taker) and therefore, the demand's optimization problem at time $t$ is the following:

$$
\max _{d_{t}} U_{t}\left(d_{t}\right)-p_{t} \times d_{t}
$$


Since $\frac{\partial U_{t}\left(d_{t}\right)}{\partial d_{t}}$ is the price per unit of electricity that the elastic demand is paying for consuming $d_{t}$ units of electricity at time $t$, budget balance means that,

$$
\sum_{n \in N} p_{n, t} e_{n, t}=\frac{\partial U_{t}\left(\sum_{n \in N} e_{n, t}\right)}{\partial\left(\sum_{n \in N} e_{n, t}\right)} \times \sum_{n \in N} e_{n, t}, \forall t \in T .
$$

The left-hand side of the above equation is the total payment that the auctioneer (ISO) pays to the GenCos. The right-hand side is the amount of money the auctioneer collects from the non-strategic demand. Note that due to market clearing, $d_{t}=\sum_{n \in N} e_{n, t}$. The condition $\sum_{n \in N} \hat{e}_{n, t} \neq 0$ means the price should follow the demand-price curve,

$$
p_{t}=\frac{\partial U_{t}\left(\sum_{n \in N} e_{n, t}\right)}{\partial\left(\sum_{n \in N} e_{n, t}\right)} .
$$

Individual rationality requires that the future utility of each GenCo $n \in N$ at each time $t \in T$ is greater than its outside option, that is, the future utility each GenCo receives by not participating in the production and expansion process. Based on the definition of the cost functions, assume the outside option for not producing or expanding to be zero. If the payment GenCo $n$ receives at time $t$ is denoted by $r_{n, t}$, then individual rationality at times $t \in T$ and $t^{+} \in T$ are expressed by the following requirements.

$$
\sum_{\tau=t, t+1, \ldots, T} \beta^{\tau}\left(-C_{n}^{e}\left(e_{n, \tau}\right)-C_{n}^{x}\left(\Delta x_{n, \tau}\right)+r_{n, \tau}\right) \geq 0 \quad \forall h_{t} \in H_{t}
$$

and

$$
\begin{array}{r}
\beta^{t}\left(-C_{n}^{e}\left(e_{n, t}\right)+r_{n, t}\right)+\sum_{\tau=t+1, t+2, \ldots, T} \beta^{\tau}\left(-C_{n}^{e}\left(e_{n, \tau}\right)-C_{n}^{x}\left(\Delta x_{n, \tau}\right)\right. \\
\left.+r_{n, \tau}\right) \geq 0, \forall h_{t^{+}} \in H_{t^{+}},
\end{array}
$$

respectively.

Note that price efficiency in this setup means that

$$
p_{n, t}=C_{n}^{e}\left(e_{n, t}\right) \quad \text { if } 0<e_{n, t}<x_{n, t}
$$

If $e_{n, t}=x_{n, t}$ then the $p_{n, t} \geq C_{n}^{e}\left(x_{n, t}\right)$ and if $e_{n, t}=0$ then $p_{n, t} \leq C_{n}^{e}(0)$.

\subsection{Design/Mechanism}

In this section, we describe how to design a mechanism that implements, in SPNE, the social welfare function of Problem (37), and in addition, is individually rational, budgetbalanced, and price efficient. In Problem (37), individual constraints exist, Equations (39) and (40), homogeneous joint constraints, Equation (41), and non-homogeneous joint constraints, Equation (42), describe the carbon emission limit. Since Equations (41) and (42) in terms of productions at time $t$, in order to decompose them into separate optimization problems, we introduce carbon permits as auxiliary variables are introduced. Also, carbon permit are designed in a way to achieve a homogeneous joint constraint for these auxiliary variables (note that Equation (42) is non-homogeneous due to different carbon emission factors $\alpha_{n, t}$ among GenCos).

Let auxiliary variable $b_{n, t}$ be GenCo $n^{\prime}$ s carbon emission permit at time $t$; then Equation (42) can be replaced with one individual and one homogeneous joint constraint as follows

$$
\begin{aligned}
0 \leq e_{n, t} & \leq b_{n, t} / \alpha_{n, t} \\
\sum_{n \in N} b_{n, t} & \leq \bar{b}_{t} .
\end{aligned}
$$


Using the auxiliary carbon permit and the (49) and (50), the carbon constraints (42) of Problem (37) is replaced by homogeneous joint constraints of the same form as Problem (25) of Section 5. Therefore, one can use the same mechanism design methodology to obtain a mechanism for problem (37) that possesses the properties established by Theorems 7 and 8 (Note that Theorems 7 and 8 are established for the multi-period, $T$-period, problem).

\subsection{Energy-and-Capacity Design vs. Energy-Only Design}

Based on the results derived so far, two alternative market designs for implementing policy targets are presented. The first is an energy-and-capacity design, the second is an energy-only design. They are compared through an example.

Energy-and-Capacity Design: Based on the replacement of the constraints expressed in Equation (42) by the constraints of (49) and (50), one way to implement electricity policies is to design one market for each homogeneous joint constraint in the modified problem. The set of markets at each time $t$ will be the following: one elastic spot market, one inelastic capacity market, and one inelastic carbon permit market. All of these markets are auctions of divisible goods and can be designed by the method proposed in Section 3. Carbon permit market is used to implement a cap constraint, while the capacity market is for implementing a floor constraint. Therefore, carbon permit markets collect money from GenCos resulting in higher prices for electricity in the spot market compared to when the carbon permit does not exist; while the capacity market pays to the sellers resulting in lower prices in the spot market compared to when the capacity market does not exist. The time-line for this dynamic energy-and-capacity mechanism is shown in Figure 2. This design implements Problem (37) in SPNE and is price efficient, budget balanced, and individually rational.

\section{Capacity Carbon Spot

Market Market Market

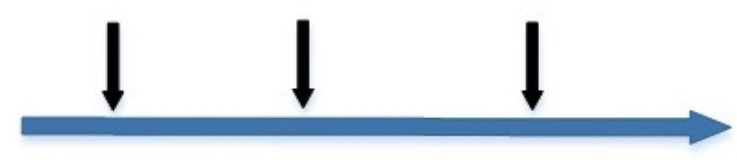

Figure 2. Timeline for the proposed energy-and-capacity mechanism.

The equilibrium price in capacity and carbon permit markets are the efficient subsidy/tax for each commodity. These prices are positive only if the corresponding constraint in Problem (37) is binding. These prices adjust automatically to technological and economic changes.

Energy-Only Design: An alternative energy-only solution for implementing reliability is to set the demand profile $\tilde{U}_{t}\left(d_{t}\right)$ above the actual demand profile $U_{t}\left(d_{t}\right)$ and, in this way, to provide incentive to GenCos for more capacity expansion [10]. The difference between the auctioned demand and the actual demand is put in the operation reserve. The advantage of this approach is that it requires a smaller number of markets in order to implement the policy targets (it does not require capacity market). However, such an approach requires more subsidy to be paid to GenCos, as shown by the following example.

Example 1 (ENERGY-ONLY VS ENERGY-AND-CAPACITY). Consider an industry modeled in Section 5 where $T=1$, i.e., a single expansion decision at time 1 is followed by a single spot market at time $1^{+}$. Consider a situation where the reliable level of capacity $\underline{x}$, the cost of expansion, $C_{n}^{x}($.$) , and the cost of production C_{n}^{e}($.$) are high but the utility of demand, U($.$) , is low. Particularly,$ assume $\underline{x}=100 \mathrm{MW}$, there are 4 Gen $\operatorname{Cos}$ with $C_{n}^{x}\left(\Delta x_{n}\right)=\Delta x_{n}^{2}, C_{n}^{e}\left(e_{n}\right)=e_{n}^{2}$ and $U(d)=10 d$. Assume further that the initial capacity $x_{0, n}$ is 0 for all $n=1,2,3,4$, and that there is no retirement payment after time $T=1$, i.e., $\eta=0 \$ / M W$. 
First consider the energy and capacity solutions. Since GenCos are similar in their costs and initial capacity, to meet the $100 \mathrm{MW}$ capacity requirement in a socially optimal way, each GenCo should expand $25 \mathrm{MW}$ capacity. This means the price for capacity in the capacity market is $50 \$$ / MW, the marginal cost of expansion by GenCos at $25 \mathrm{MW}$ capacity. Therefore, total payment by the system operator to GenCos in the capacity market is $100 \mathrm{MW} \times 50 \$ / \mathrm{MW}=5 \mathrm{k} \$ / \mathrm{MW}$. This amount is the subsidy for reliable capacity. Moreover in the spot market, the price of electricity is the marginal utility of demand which is $10 \$ / M W$. Therefore, each GenCo produces $5 \mathrm{MW}$ of electricity to have marginal cost of production equal to $10 \$ / M W$ and the total payment to GenCos for producing electricity at the spot market is $4 \times 5 \mathrm{MW} \times 10 \$ / M W=200 \$$.

Next, consider an energy-only solution. The operation reserve demand should be set high enough to ensure Gen Cos have incentive to expand $25 \mathrm{MW}$ each, $\Delta x_{n}=25 \mathrm{MW}, n=1,2,3,4$. If GenCos expand by $25 \mathrm{MW}$ each, they should use all of it in the spot market i.e., $e_{n}=25 \mathrm{MW}, n=$ $1,2,3,4$, since otherwise they do not have incentive to expand by the above amount (saturation property). Therefore, the price of electricity in the spot market should be the marginal of production and expansion together, and this is equal to $2 \times \Delta x_{n}+2 \times e_{n}=2 \times 25+2 \times 25=100 \$ / M W$. The total payment to the GenCos is $100 \$ / M W \times 100 \mathrm{MW}=10 \mathrm{k} \$$. To calculate the subsidy paid for reliable capacity in the energy-only solution, one should calculate the payment for operation reserve; this is the difference between the total payment in the spot market, 10,000\$, and the efficient payment for real demand, 200\$. Therefore, the subsidy for reliable capacity in the energy-only solution is $10,000 \$-200 \$=9800 \$$, which is almost twice as large as the $5 k \$$ efficient subsidy in the energy-and-capacity solution.

\subsection{Impact on Policy Debates}

The above solutions for electricity policy targets shed light on the electricity policy debates. First, using the auctions designed in Section 3, price efficiency is achieved without using price caps (or offer caps), market monitoring, or other sorts of market interventions. Second, both the energy-and-capacity solution/market as well as an energy-only solution/market achieve planning reserve requirements. The energy-and-capacity solution achieves efficient subsidy (by using the decomposition technique in Section 5), but requires extra markets (capacity markets) and corresponding regulations. The energy-only solution requires more information and complex computations by the system operator, and pays more subsidy to GenCos compared to the energy-and-capacity solution. Note that the results in Section 4 show that in the absence of planning reserve requirements, no capacity markets, or other subsidiary mechanisms are required to achieve socially optimal investment (this is in contrast to References [11,12]). Finally, by using the decomposition technique in Section 5, the carbon market finds the price of carbon as the efficient taxation required to meet the carbon emission target.

\section{Implementation}

The market mechanisms presented in this paper were developed under the assumption that GenCos have complete (symmetric) information about the environment (see Section 1.5 for the use and implications of this assumption in the electricity industry). Therefore, each GenCo can calculate the subgame perfect Nash equilibrium of the game induced by the market mechanism according to Theorems 7 and 8 . Note that finding these equilibrium strategies requires solving Problem (37); this problem can be solved by dynamic programming (each GenCo can solve the dynamic program). Similar to other sustainable practices, the implementation can benefit from Industry standards $[80,80]$ using advance analytic and state-of-the-art machine learning for solving the optimization problems [81-83]. Also, the auctions presented here require indexing of the GenCos and paying each GenCo according to the bids of the next one, but this indexing can be relaxed in alternative designs with similar properties as discussed at the end of Section 3.3. 


\section{Model Limitations and Possible Extensions}

The model presented in Section 2 includes the essential features needed for the study of electricity policy targets, but of course does not capture all the salient features of real-world electricity markets; nevertheless, it can be extended in the following major directions so as to become closer to reality. This can be achieved by including (i) the network constraints, (ii) delays in building new capacities, (iii) fixed (startup) costs, (iv) information asymmetries across GenCos, and v) other engineering constraints such as ramping and $n-1$ reliability constraints. The impact of such extensions on the design of electricity markets is further discussed in Section 9.

The first extension is related to the network. This study did not consider the network constraints in the model so as to focus on market forces and the effect of policy constraints, Equations (39)-(42), on the strategic GenCos' production and capacity expansion plans. This assumption is adapted in other major studies of wholesale electricity markets that are primarily for finding the time difference of the price (see [84] for survey). In practice, some pooling markets like Germany and France do not consider the network constraints for clearing the wholesale pooling market; the challenging optimal power flow problem is then solved at the re-dispatch phase to slightly modify the trades for a feasible dispatch of electricity in the network. Even in the cases when the network constraints are considered in clearing the wholesale market, these constraints have minor impact in the market performance when the network is not congested and the locational marginal prices are close to each other, for example in California [85]. Reference [73] studies electricity market design considering network constraints while incorporating loop flow effect to the second order approximation and shows their local public good effect.

The second extension relates to capacity expansion. This study assumes that the new capacities are immediately built and available at the same year; in practice it takes a number of years to build and add them to the system. Also, the model does not consider depreciation of the capacity. The above modeling assumptions are made for simplicity of presentation; the results of this paper hold also when newly planned capacities are added several years later, and when there exists a fixed constant rate or an uncertain reliable rate of capacity depreciation. In addition, whereas in practice companies review their expansion decisions each year, there are numerous spot markets during the year (one in almost every 10 minutes). Therefore, the spot market in this model should be interpreted as an abstract market model that captures the aggregate effect of all the spot markets during a year. The results of the paper hold if several spot markets are included in between the capacity expansions. Finally, this study has not considered technology selection by the GenCos in their expansion, rather their decisions are limited to the size of expansion. Adding diverse technology for GenCos does not change the results, but GenCos will consider the emission factor of each technology into account.

Next, one can revisit the assumptions on costs and utilities. Although this study assumed costs of production and expansion are convex, in real world both production and expansion can have economy of scale and therefore can be concave [86]. Also although this study assumed the costs are zero for zero production and expansion, in reality there exist start-up costs or fixed costs.

Another major extension of the model is related to the information asymmetry that exists among the strategic GenCos. This study has assumed information is symmetric, but in reality, the GenCos have private information on their costs, technology, and budgets. The assumption of symmetric information among GenCos and the use of Nash equilibrium as a solution concept is central to the theoretical results. This assumption has both theoretical and practical reasons. Theoretically, eliminating information asymmetry and its corresponding information rent allows us to develop a basic understanding of the problems and rigorous analytical results. This assumption is made in majority of the literature (for example see [87]). A number of papers argue that symmetric information is a realistic assumption for electricity markets. For example, Reference [88] argues that symmetric information of the bidders (GenCos), which is superior to the information of 
the auctioneer (system operator), is a common rationale and a reasonable assumption for oligopolistic markets where bidders monitor each other (each others' technologies and capacity), but the auctioneer does not have access to such information.

Other than the above issues, the model does not capture transmission expansion planning and new entry. Since the model does not include network constraints, adding transmission expansion is not readily available. However, it should be noted that in a model with network constraints, the main challenge of adding transmission expansion decisions will be in the case where the productions and prices in electricity market can impact the transmission expansion outcomes and therefore the GenCo's should consider this impact in their strategic bidding. Otherwise, if the network expansion is determined exogenously and independently to the decision of the GenCos, it can be simply added to the information available to the GenCos. New entry of GenCos can be readily added to the model. If new entry to the industry is feasible, new GenCos will enter until the profit of the last GenCo who enters the market is equal to the outside investment options. Such constraint will determine the number of the GenCos in the economy at equilibrium.

Finally, there exist other constraints in electricity networks such as ramping constraints and $n-1$ power flow constraints for short term reliability. These constraints have not been considered in the model as this study primarily focuses on long-term investment constraints.

\section{Conclusions and Future Directions}

This study proposed a model for the restructured electricity industry that captures all policy targets and takes into account the technological changes in the industry. Using this model, this study developed a methodology based on mechanism design and auction design with constraints that achieves all electricity policy targets. To the best of our knowledge, this paper presents the first attempt to address all electricity policy targets simultaneously using a concrete stylized mathematical model along with a methodological approach.

Future work related to the model includes: (i) network constraints and transmission expansion, (ii) delays in building new capacities, (iii) fixed (startup) costs, (iv) information asymmetries across GenCos, (v) other engineering constraints such as ramping, (vi) new GenCo entries, and (vii) a broader definition of reliability by considering a broader definition of reliability by considering alternative constraints for it such as the $n-1$ criterion, interconnection frequency response, element availability percentage, etc. Future work related to the proposed methodology includes the design of: (i) coalition free mechanisms [26], (ii) auctions with a more extensive set of individual and joint constraints that capture alternative interpretations of reliability (such as the $n-1$ criterion), (iii) auctions where a divisible good is allocated among an oligopoly of agents/firms and the auctioneer (Section 3), (iv) supply chains where producers are competing in the end-product market to meet the demand, but there is no competition on the other upstream goods/resources in the supply chain (Section 4) [89,90], and (v) oligopolies where agents interact with one another over a time horizon and have to make a certain level of excess investment because of the possible occurrence of unexpected events, such as demand shocks or supply disruption, over the horizon (this is the case in other critical sectors such as natural gas and oil-refining) (Section 5).

Author Contributions: Conceptualization, M.R. and D.T.; Formal analysis, M.R. and D.T.; Investigation, M.R. and D.T.; Methodology, M.R. and D.T.; Supervision, D.T.; Writing—original draft, M.R.; Writing-review \& editing, M.R. and D.T. All authors have read and agreed to the published version of the manuscript.

Funding: This research was supported in part by NSF Grant CNS-1238962 and ARO Grant W911NF17-1-0232. 
Acknowledgments: The authors would like to thank William Hogan, Paul Milgrom, Robert Wilson, Asuman Ozdaglar, Ian Hiskens, Mohammad Akbarpour, Shaowei Ke, and Ramesh Johari for useful discussions.

Conflicts of Interest: The authors declare no conflict of interest.

\section{Appendix A. Proof of Properties for the Proposed Elastic and Inelastic Auctions}

Appendix A.1. Preliminaries

A set of messages $m^{*}=\left(m_{1}^{*}, m_{2}^{*}, \ldots, m_{N}^{*}\right)$ is an NE of the game induced by the proposed mechanism if for each producer $n \in N, m_{n}^{*}=\left(\hat{e}_{n}^{*}, p_{n}^{*}\right)$ is a best response to the others' messages denoted by $m_{-n}^{*}$.

First consider the auction with elastic demand. The pair $\left(\hat{e}_{n}^{*}, \hat{p}_{n}^{*}\right)$ is producer $n^{\prime}$ s best response to other producers' messages, if it is a solution to their profit maximization problem

$$
\begin{aligned}
\left(\hat{e}_{n}^{*}, \hat{p}_{n}^{*}\right)= & \arg \max _{\hat{e}_{n}^{*} \in \mathbb{R}, \hat{p}_{n}^{*} \in \mathbb{R}^{+}}\left\{-C_{n}^{e}\left(\hat{e}_{n}\right)+r_{n}^{\text {elas. }}\right\} \\
\text { s.t. } & \hat{p}_{n} \geq 0 .
\end{aligned}
$$

Problem (A1) is a strictly concave optimization (the Hessian of $\hat{p}_{n}^{-0.5}$ zelas. $_{n}^{2}$ is positive semidefinite in the domain).

Since both Problems (4) and (A1) are strictly concave optimization problems, they have a unique solution, and the Karush-Kuhn-Tucker (KKT) conditions are necessary and sufficient for optimality. The Lagrangian for Problem (4) is

$$
L_{(4)}=U\left(\sum_{n \in N} e_{n}\right)-\sum_{n \in N} C_{n}^{e}\left(e_{n}\right)
$$

and the KKT conditions are, $\forall n \in N$,

$$
\left.\frac{\partial U\left(\sum_{n \in N} e_{n}\right)}{\partial e_{n}}\right|_{e^{*}}-\left.\frac{\partial C_{n}^{e}}{\partial e_{n}}\right|_{e^{*}}=0 .
$$

The Lagrangian for Problem (A1) is

$$
L_{\hat{Q}_{1, n}}=-C_{n}^{e}\left(\hat{e}_{n}\right)+r_{n}^{\text {elas. }}-\theta_{n} p_{n},
$$

and the corresponding KKT conditions are

$$
\begin{aligned}
-\left.\frac{\partial C_{n}^{e}}{\partial \hat{e}_{n}}\right|_{m^{*}}+\left.\frac{\partial r_{n}^{\text {elas. }}}{\partial \hat{e}_{n}}\right|_{m^{*}} & =0, \\
\left.\frac{\partial r_{n}^{\text {elas. }}}{\partial \hat{p}_{n}}\right|_{m^{*}}-\hat{\theta}_{n}^{*} & =0, \\
\hat{\theta}_{n}^{*} \hat{p}_{n}^{*} & =0, \\
\hat{\theta}_{n}^{*} & \geq 0 .
\end{aligned}
$$

Next we consider the auction for inelastic demand. Let $m^{*}=\left(\hat{e}_{1}^{*}, \hat{e}_{2}^{*}, \ldots, \hat{e}_{N}^{*}, \hat{p}_{1}^{*}, \hat{p}_{2}^{*}, \ldots, \hat{p}_{N}^{*}\right)$ be an NE of the game induced by the mechanism. Then, $m^{*}$ is a solution to every producer's profit maximization problem, that is, $\forall n \in I$

$$
\begin{gathered}
\left(\hat{e}_{n}^{*}, p_{n}^{*}\right)=\arg \max _{\hat{e}_{n}, \hat{p}_{n}}-C_{n}^{e}\left(\hat{e}_{n}\right)+r_{n}^{\text {inelas. }} \\
\text { s.t } \quad \hat{p}_{n} \geq 0 .
\end{gathered}
$$


Problem (A10) is a strictly concave optimization. Since both Problems (5) and (A10) are strictly concave optimization, they have a unique solution and the KKT conditions are necessary and sufficient for optimization. The Lagrangian for Problem (5) is

$$
\left.L_{(5)}=-\sum_{i \in I} C_{n}^{e} e_{n}\right)+\kappa\left(\sum_{n \in N} e_{n}-\underline{d}\right)
$$

and KKT conditions are

$$
\begin{aligned}
\left.\frac{\partial C_{n}^{e}}{\partial e_{n}}\right|_{e^{*}}-\kappa^{*} & =0 \quad \forall n \in N, \\
\kappa^{*}\left(\sum_{n} e_{n}^{*}-\underline{d}\right) & =0, \\
\kappa^{*} & \geq 0 .
\end{aligned}
$$

The Lagrangian for Problem (A10) is

$$
L_{\hat{Q}_{n}^{\text {inelas. }}}=-C_{n}^{e}\left(\hat{e}_{n}\right)+r_{n}^{\text {inelas. }}+\hat{\theta}_{n} \hat{p}_{n}
$$

and the KKT conditions are, $\forall n \in N$,

$$
\begin{aligned}
-\left.\frac{\partial C_{n}^{e}}{\partial \hat{e}_{n}}\right|_{\mathbf{m}^{*}}+\left.\frac{\partial r_{n}^{\text {inelas. }}}{\partial \hat{e}_{n}}\right|_{\mathbf{m}^{*}} & =0, \\
\left.\frac{\partial r_{n}^{\text {inelas. }}}{\partial p_{n}}\right|_{\mathbf{m}^{*}}+\hat{\theta}_{n}^{*} & =0, \\
\hat{\theta}_{n}^{*} & \geq 0 .
\end{aligned}
$$

Appendix A.2. Proof of Results for the Proposed Elastic and Inelastic Auctions

Proof of Lemma 1. First, consider the auction for elastic demand. Assume $\zeta_{n}^{\text {elas. }}{ }^{*} \neq 0$ at NE for some $n \in N$. Then, the price at equilibrium, $\hat{p}_{n}^{*}$, must be strictly positive because if it is zero, the GenCo $n$ 's utility at equilibrium is equal to $-\infty$ and agent $n$ can improve its utility by changing $\hat{p}_{n}$ to a positive value. From Equations (A7), we have

$$
\left.\frac{\partial u_{n}}{\partial \hat{p}_{n}}\right|_{m^{*}}=\left.\frac{\partial r_{n}^{\text {elas. }}}{\partial \hat{p}_{n}}\right|_{m^{*}}=-\left.0.5 \hat{p}_{n}^{*}{ }_{n}^{-1.5} \zeta_{n}^{\text {elas. }}{ }^{* 2}\right|_{m^{*}}=0 .
$$

Since $\hat{p}_{n}^{*}{ }_{n}^{-1.5}>0$, we should have $\zeta_{n}^{\text {elas. }}=\left(D\left(\hat{p}_{n+1}\right)-\sum_{n \in N} \hat{e}_{n}^{*}\right)^{2}=0$, which contradicts the assumption of $\zeta_{n}^{\text {elas. }}{ }^{*} \neq 0$.

Next, consider the auction for inelastic demand. Since the equilibrium is non-trivial all prices cannot be zero at the same time, therefore, either all prices are positive or there exists a zero price followed by a positive price.

Case 1: For all $n \in N, \hat{p}_{n}^{*}>0$.

Here, $\hat{p}_{n}^{*} \geq 0$ is not binding. Therefore, at NE for all $n \in N$,

$$
\begin{array}{r}
\left.\frac{\partial \hat{u}_{n}}{\partial \hat{p}_{n}}\right|_{m^{*}}=\left.\frac{\partial r_{n}^{\text {inelas. }}}{\partial \hat{p}_{n}}\right|_{m^{*}}= \\
-2\left(\hat{p}_{n}^{*}-\hat{p}_{n+1}^{*}\right)-2 \zeta^{\text {inelas. }^{* 2}}=0 .
\end{array}
$$


Summing up Equation (A21) over all $n$ we get:

$$
\begin{aligned}
& \left.\sum_{n \in N} \frac{\partial r_{n}^{\text {inelas. }}}{\partial \hat{p}_{n}}\right|_{m^{*}} \\
& =\sum_{n \in N}\left[-2\left(\hat{p}_{n}^{*}-\hat{p}_{n+1}^{*}\right)-2 \zeta^{\text {inelas. }{ }^{* 2}}\right] \\
& =-2 N \zeta^{\text {inelas. }{ }^{* 2}}=0 .
\end{aligned}
$$

The last equality is achieved if and only if $\zeta_{t}^{\text {inelas. }}{ }^{*}=0$, i.e., if and only if $\sum_{n \in N} \hat{e}_{n}^{*}=\underline{d}$. Case 2: $\exists n \in N$ s.t. $\hat{p}_{n}^{*}=0$ and $\hat{p}_{n+1}>0$.

We prove that this case cannot exist. Since the constraint $\hat{p}_{n} \geq 0$ is binding for $\hat{p}_{n}^{*}=0$, at NE we must have

$$
\left.\frac{\partial \hat{u}_{n}}{\partial \hat{p}_{n}}\right|_{m^{*}} \leq 0
$$

Furthermore, using $\hat{p}_{n}^{*}=0$ we obtain

$$
\left.\frac{\partial \hat{u}_{n}}{\partial \hat{p}_{n}}\right|_{m^{*}}=2\left[\hat{p}_{n+1}^{*}-\zeta^{\text {inelas. }}{ }^{* 2}\right] \leq 0 .
$$

Since $\hat{p}_{n+1}^{*}>0$, because of Equation (A21),

$$
\left.\frac{\partial \hat{u}_{n+1}}{\partial \hat{p}_{n+1}}\right|_{m^{*}}=2\left(\hat{p}_{n+2}^{*}-\hat{p}_{n+1}^{*}\right)-2 \zeta^{\text {inelas. }}{ }^{* 2}=0
$$

From (A25) it follows that

$$
\hat{p}_{n+2}^{*}=\hat{p}_{n+1}^{*}+\zeta^{\text {inelas. }}{ }^{* 2} \geq \hat{p}_{n+1}^{*}>0 \text {. }
$$

Since $\hat{p}_{n+2}^{*}>0$, we can repeat the same argument as Equations (A25) and (A26) to calculate $\hat{p}_{n+3}^{*}$ and show it is positive. Continuing the same argument on the sequence of the prices $\left\{\hat{p}_{n+3}^{*}, \hat{p}_{n+4}^{*}, \ldots, \hat{p}_{n}^{*}, \hat{p}_{1}^{*}, \ldots, \hat{p}_{n-1, t}^{*}, \hat{p}_{n}^{*}\right\}$, we obtain

$$
\hat{p}_{n}^{* 2}=\hat{p}_{n+1}^{*}+N \zeta^{\text {inelas. }}{ }^{* 2}>0 .
$$

This contradicts the assumption of $\hat{p}_{n}^{*}=0$.

Proof of Theorem 1. First consider the auction for elastic demand. From Equation (14) of Lemma 1, we have

$$
D\left(\hat{p}_{n}^{*}\right)=\sum_{j \in N} \hat{e}_{j}^{*} \quad \forall n \in N
$$

As a direct consequence of Equation (A28),

$$
\hat{p}_{n}^{*}=\hat{p}^{*}=U^{\prime}\left(\sum_{j \in N} e_{j}^{*}\right)>0 \quad \forall n \in N .
$$

The inequality in Equation (A29) follows from concavity of demand. Moreover, Equations (9), (14) and (16) imply $r_{n}^{*}=\hat{p}^{*} \hat{e}_{n}^{*}$, and because $\zeta_{n}^{\text {elas. }}{ }^{*}=0$,

$$
\left.\frac{\partial r_{n}^{\text {elas. }}}{\partial \hat{e}_{n}}\right|_{m^{*}}=\hat{p}^{*}-\left.2 \hat{p}^{*}{ }^{0.5} \zeta_{n}^{\text {elas. }} * \frac{\partial \zeta_{n}^{\text {elas. }}}{\partial \hat{e}_{n}}\right|_{m^{*}}=\hat{p}^{*}
$$

From Equations (A6) and (A30), we have

$$
C^{\prime}\left(e_{n}^{*}\right)=p^{*}
$$


Next, consider the auction with inelastic demand. First note that from proof of Lemma 1, for any non-trivial NE, we have $p_{n}^{*}>0, \forall n \in N$. Using Equation (15) in Equation (A21), we obtain

$$
\left.\frac{\partial r_{n}^{\text {inelas. }}}{\partial \hat{p}_{n}}\right|_{m^{*}}=-2\left(\hat{p}_{n}^{*}-\hat{p}_{n+1}^{*}\right)=0 .
$$

Therefore,

$$
\hat{p}_{n}^{*}=\hat{p}_{n+1}^{*}=\hat{p}^{*} \quad \forall n \in N .
$$

Next, from Lemma 1, we get $\left[\sum_{n \in N} \hat{e}_{n}^{*}-\underline{d}\right]=0$, therefore,

$$
\hat{p}^{*}\left[\sum_{n \in N} \hat{e}_{n}^{*}-\underline{d}\right]=0 \quad \forall n \in N .
$$

Furthermore, Equations (9), (15) and (A33) imply

$$
r_{n}^{\text {inelas. }}{ }^{*}=\hat{p}^{*} \hat{e}_{n}^{*}
$$

and

$$
\left.\frac{\partial r_{n}^{\text {inelas. }}}{\partial \hat{e}_{n}}\right|_{m^{*}}=\hat{p}^{*}-\left.2 \hat{p}^{*} \zeta^{\text {inelas. }} \frac{\partial \zeta^{\text {inelas. }}}{\partial \hat{e}_{n}}\right|_{m^{*}}=\hat{p}^{*} .
$$

Finally, using Equation (A36) in Equation (A17) of the KKT condition, we obtain

$$
\hat{p}^{*}=C_{n}^{e \prime}\left(\hat{e}_{n}^{*}\right) .
$$

Proof of Theorem 2. At the trivial equilibrium amount paid to the GenCos is zero. Therefore, the auctions are budget balanced. We discuss the case of non-trivial equilibria below.

First consider the auction with elastic demand. At equilibrium, producer $n$ receives $r_{n}^{\text {elas. }}{ }^{*}=p^{*} e_{n}^{*}$ and demand pays $\hat{p}^{*} \sum_{n \in N} \hat{e}_{n}^{*}=\sum_{n \in N} r_{n}^{\text {elas. }}{ }^{*}$ where from Equation (A29), $p^{*}=\left(U^{-1}\right)^{\prime}\left(\sum_{n \in N} e_{n}^{*}\right)$; therefore, the sum of all payments adds up to zero. For the auction with inelastic demand, the amount charged to producer, $\sum_{n \in N} r_{n}^{\text {elas. }}{ }^{*}$, can be collected from the inelastic demand without changing the behavior of the demand. Therefore, the auction is budget balanced.

Proof of Theorem 3. To show that the allocation $e_{n}^{*}=\hat{e}_{n}^{*}, n \in N$, corresponding to nontrivial equilibrium $m^{*}$ is a solution of Problem (4) (correspondingly Problem (5)), we specify the KKT parameters of Problem (4) (correspondingly Problem (5)) based on the KKT parameters of Problem (A1) (correspondingly Problem (A10)) as follows.

First consider the auction for elastic demand. Let $m^{*}$ be an NE of the game induced by the proposed mechanism ( $\hat{p}_{n}^{*}>0, \quad \forall n \in N$ by Theorem 1). Then, Equations (A6)-(A8) along with Theorem 1 show that Equation (A4) is satisfied for all $n \in N$. Consequently, $\left(\hat{e}_{1}^{*}, \hat{e}_{2}^{*}, \ldots, \hat{e}_{N}^{*}\right)$ is a solution of Problem 4 .

Second, consider the auction for inelastic demand. Let $m^{*}$ be a non-trivial NE of the game induced by the mechanism. Then, $m^{*}$ is a solution to every GenCo's profit maximization problem. We set

$$
e_{n}^{*}=\hat{e}_{n}^{*}, \quad \kappa^{*}=\hat{p}^{*} \quad \forall n \in N .
$$

Then, Equations (A17)-(A19) along with Theorem 1 show that Equations (A13)-(A14) are satisfied.

Proof of Theorem 4. We show that each producer $n \in N$ weakly prefers the equilibrium outcome to the outside option $\left(e_{n}, r_{n}\right)=(0,0)$ resulting when producer $n$ does not participate in the auction. 
From Equation (17), we obtain that GenCo n's utility at equilibrium is

$$
-C_{n}^{e}\left(\hat{e}_{n}^{*}\right)+r_{n}^{\text {elas.* }}=-C_{n}^{e}\left(\hat{e}_{n}^{*}\right)+C_{n}^{e^{\prime}}\left(\hat{e}_{n}^{*}\right) \times \hat{e}_{n}^{*} .
$$

Furthermore, for the convex and increasing function $C_{n}^{e}($.$) with C_{n}^{e}(0)=0$ we have

$$
C_{n}^{e}\left(e_{n}\right) \leq C_{n}^{e^{\prime}}\left(e_{n}\right) \times e_{n} \quad \text { for any } \quad e_{n} \geq 0 .
$$

Combining (A39) and (A40) we get,

$$
u_{n}\left(m^{*}\right)=-C_{n}^{e}\left(\hat{e}_{n}^{*}\right)+C_{n}^{e^{\prime}}\left(\hat{e}_{n}^{*}\right) \hat{e}_{n}^{*} \geq 0 .
$$

Proof of Lemma 2. We first prove the existence of a unique trivial equilibrium for the inelastic demand. Consider GenCo $n$ and assume all other GenCos propose a price of zero; we prove GenCo $n$ 's best response is to bid zero price and zero quantity. Since $\hat{p}_{n+1}^{*}=0$, GenCo $n$ is paid no compensation for his production, i.e., $\hat{p}_{n+1}^{*} e_{n}=0$. Other terms in GenCo $n$ 's utility function include penalty term and cost of production. The penalty terms are non-positive for any $\hat{p}_{n}>0$ and zero for $\hat{p}_{n}=0$. Therefore, producer $n$ maximizes this utility by proposing $\hat{p}_{n}=0$. Also, the cost of production is positive for all $\hat{e}_{n} \neq 0$ and zero at $\hat{e}_{n}=0$. Therefore, GenCo $n^{\prime}$ s best response is $e_{n}=0$.

For elastic demand, the proof of Lemma 1 and Theorem 3 is valid at all equilibria. Therefore, in the case where the solution of Problem (4) is such that $\exists n \in N: e_{n}^{*}>0$ there does not exist any trivial equilibrium. This is because from Theorem 1, GenCos' price proposals are $\hat{p}^{*}=C_{n}^{e^{\prime}}\left(e_{n}^{*}\right)$, which is greater than zero. On the other hand, if the solution of Problem (4) satisfies $e_{n}^{*}=0, \forall n \in N$ then the price proposals are also zero and the game induced by the mechanism has a unique equilibrium which is trivial.

\section{Appendix B. Extension of Results of Proposed Auctions with Joint Constraints to Long-Term Interactions Among Strategic GenCos with Symmetric Uncertainty}

In this Section, we extend the result of Section 5 to a multi-period model.

\section{Appendix B.1. Model Extension}

Consider the extended reliability targets in the form of the following planning reserve requirements:

$$
\sum_{n \in N} x_{n, t} \geq \underline{x}_{t}\left(\mathbf{w}_{t-1}\right), \quad \forall t \in T .
$$

This means that at each time $t$, there is a minimum capacity requirement. The expected social welfare maximizing investment and production is

$$
\begin{array}{ll} 
& \max _{\sigma_{n, t} \in \Sigma_{n, t}, \gamma_{n, t} \in \Gamma_{n, t}: n \in N, t \in T} E_{\mathbf{w}_{1: T}}\left[\sum_{t \in T} \beta^{t} U_{t}\left(\sum_{n \in N} e_{n, t}, \mathbf{w}_{t}\right)-\right. \\
& \left.\sum_{t \in T} \sum_{n \in N} \beta^{t}\left\{C_{n, t}^{e}\left(e_{n, t}, \mathbf{w}_{t}\right)+C_{n, t}^{x}\left(\Delta x_{n, t}, \mathbf{w}_{t-1}\right)\right\}+\beta^{T+1} \sum_{n \in N} \eta\left(\mathbf{w}_{T}\right) x_{n, T}\right] \\
\text { s.t. } \quad & 0 \leq e_{n, t} \leq x_{n, 0}+\sum_{\tau=1, \ldots, t} \Delta x_{n, \tau} \\
& 0 \leq \Delta x_{n, t} \\
& \sum_{n \in N} x_{t} \geq \underline{x_{t}}\left(\mathbf{w}_{t-1}\right)
\end{array}
$$

In Lemma 3, we prove that the solution of Problem (A43), the expansion strategies at time $t, \sigma_{n, t}^{*}$, are only functions of $S_{t}=\left\{x_{t-1}, w_{1: t-1}\right\}$ and generation strategies at time $t^{+}$, 
$\gamma_{n, t}^{*}$, are only functions of $S_{t^{+}}=\left\{x_{t}, w_{1: t}\right\}$. In other words, $S_{t}$ and $S_{t^{+}}$are information state for Problem (A43). We will also prove that the same is true for the GenCo's SPNE strategy in the game induced by the mechanism we design.

\section{Appendix B.2. Design/Mechanism}

Using dynamic programming and value function, Problem (A43) can be decomposed into a set of sequential optimization problems, one for each year (See Appendix B.4). Furthermore, using the decomposition technique of Section 5.2, the optimization of each year $t \in T$ can be further decomposed into two optimization problems, one for capacity expansion and one for electricity generation. Considering this decomposition, we use $2 \times T$ markets for implementing the corresponding outcome: each year $t \in T$ has a capacity market for auctioning the inelastic capacity $\left(\underline{x}_{t}\left(\mathbf{w}_{t-1}\right)-\sum_{n \in N} x_{n, t-1}\right)$, followed by an elastic spot market for auctioning electricity demand with utility $U_{t}\left(d_{t}, w_{t}\right)$.

\section{Appendix B.3. Properties of the Mechanism}

In the game induced by the energy-and-capacity mechanism, the strategy of each seller $n$ consists of both strategies $\tilde{\sigma}_{n, t}, t \in T$ determining their bids in the capacity market and strategies $\tilde{\gamma}_{n, t^{+}}, t \in T$ determining their bids in the spot markets. The history of the game at the time of expansion is

$$
\begin{aligned}
\tilde{h}_{t} & =\left\{x_{0}, \Delta \tilde{x}_{1: t-1}, \tilde{p}_{1: t-1}, \hat{e}_{1: t-1}, \hat{p}_{1: t-1}, w_{1: t-1}\right\} \in \tilde{H}_{t} \\
\tilde{H}_{t} & :=\left\{x_{0}, \Delta \tilde{x}_{1: t-1}, \tilde{P}_{1: t}, \hat{E}_{1: t-1}, \hat{P}_{1: t-1}, w_{1: t-1}\right\}
\end{aligned}
$$

And at the spot market $t$ it is

$$
\begin{aligned}
\tilde{h}_{t^{+}} & =\left\{x_{0}, \Delta \tilde{x}_{1: t}, \tilde{p}_{1: t}, \hat{e}_{1: t-1}, \hat{p}_{1: t-1}, w_{1: t}\right\} \\
\tilde{H}_{t^{+}} & =\left\{x_{0}, \Delta \hat{x}_{1: t}, \tilde{P}_{1: t}, \hat{E}_{1: t-1}, \hat{P}_{1: t-1}, w_{1: t}\right\}
\end{aligned}
$$

Therefore, $\forall n \in N$,

$$
\begin{array}{r}
\tilde{\sigma}_{n, t}: \tilde{H}_{t} \rightarrow\left[0, \overline{\Delta x}_{n, t}\right] \times \mathbb{R}_{+} \\
\tilde{\gamma}_{n, t^{+}}: \tilde{H}_{t^{+}} \rightarrow\left[0, x_{n, t}\right] \times \mathbb{R}_{+}
\end{array}
$$

We denote the set of all such strategies by $\tilde{\Sigma}_{n, t}$ and $\tilde{\Gamma}_{n, t}$.

The properties of the proposed energy-only economy at its SPNE are described by Lemma 3 and Theorem 9.

We first show that there exists SPNE strategies of the game induced by the energyand-capacity mechanism that are only a function of the payoff relevant information i.e., they are Markov.

Lemma 3 (MARKOV STRATEGIES). There is a solution of Problem (A43) where the expansion (respectively production) strategies are functions of $S_{t}=\left\{x_{1: N, t-1}, w_{1: t-1}\right\}$ (respectively $S_{t^{+}}=$ $\left\{x_{1: N, t}, w_{1: t}\right\}$ ). There is an SPNE induced by the proposed finite horizon mechanism where the GenCos' expansion (respectively production) strategies are a function of $S_{t}$ (respectively $S_{t^{+}}$).

Lemma 3 implies the following corollary.

Corollary 1 (MYOPIC BEHAVIOR). There is an SPNE where GenCos act myopically in the spot market.

This is because the actions of the GenCos in the spot market at time $t^{+}$, production, and price proposals, do not change the information state $S_{\tau}$ and $S_{\tau^{+}}$for $\tau>t$ i.e., these actions do not have any effect in the future strategies of the agents. Therefore, it is best for GenCos to act myopically and maximize their utility in the current spot market. 
We call an SPNE of the game induced by the mechanism non-trivial, if the equilibrium price proposal in the capacity markets of time $t, p_{t}^{c *}$ is not zero for all $t \in T$.

Theorem 9 (EFFICIENCY). There is a unique non-trivial SPNE that Pareto dominates all the trivial SPNEs of the game induced by the mechanism of Appendix B.2 and has the following features.

(i) (SOCIAL OPTIMALITY) The expansion and production outcomes corresponding to the unique non-trivial SPNE are socially optimal, that is they are equal to the solution of Problem (A43).

(ii) (PRICE EFFICIENCY) For all $t \in T$, there is a unique capacity price proposal, $p_{t}^{c *}$ in the capacity market, and a unique electricity price, $p_{t}^{*}$ in the spot market for all GenCos $n \in N$, which are efficient, that is

$$
\begin{aligned}
p_{t}^{*} & =C^{\prime}\left(e_{n, t}^{*}\right) \text { if } 0<\hat{e}_{n, t}^{*}<x_{n, t}, \\
p_{t}^{c *} & =C^{x \prime}\left(\Delta x_{n, t}^{*}\right)-E_{\mathbf{w}_{t} \mid S_{t}}\left\{\left.\frac{\partial V_{n, t^{+}}\left(x_{t^{+}}, w_{1: t-1}, \mathbf{w}_{t}\right)}{\partial \Delta x_{n, t}}\right|_{m_{t}^{*}}\right\} \text { if } 0<\Delta x_{n, t}^{*}<\overline{\Delta x}_{n, t},
\end{aligned}
$$

where $V_{n, t^{+}}\left(S_{t^{+}}\right)$is the future expected revenue of producer $n$ from time $t^{+}$on.

(iii) (BUDGET BALANCED) All the capacity and spot markets are budget balanced at equilibrium. For the elastic spot market at time $t \in T$,

$$
\hat{p}_{t}^{*}=\frac{\partial U_{t}\left(\sum_{n \in N} e_{n, t}, w_{t}\right)}{\partial\left(\sum_{n \in N} e_{n, t}\right)} .
$$

if $\sum_{n \in N} e_{n}>0$

(iv) (INDIVIDUAL RATIONALITY) The proposed mechanism is individually rational, that is for every time $t \in T$ and realization of the history, the sum of the current and expected future utility is non-negative.

Appendix B.4. Proof of Lemma 3 and Theorem 9

Proof of Lemma 3. First we consider Problem (A43). This is a constraint Markov decision process (MDP) with constraints on the GenCos' capacity expansions and limits on the GenCos' production. Therefore there exist optimal Markov decision strategies for GenCos' expansion and production. These strategies are determined by the following dynamic programming. Time $T+1$

$$
V_{T+1}\left(s_{T+1}\right)=\eta\left(w_{T}\right) \sum_{n \in N} x_{n, T+1} .
$$

Problem $Q_{t^{+}}^{3}$

At time $t^{+}$optimal generations are according to the following optimization problem,

$$
\begin{aligned}
V_{t^{+}}\left(X_{t^{+}}, w_{1: t}\right) & =\max _{e_{n, t}, n \in N} U_{t}\left(\sum_{n \in N} e_{n, t}, w_{t}\right)-\sum_{n \in N} C_{n, t}^{e}\left(e_{n, t}, w_{t}\right)+\beta V_{t+1}\left(X_{t^{+}}, w_{1: t}\right) \\
\text { s.t. } & 0 \leq e_{n, t} \leq x_{n, t^{+}} \quad \forall n \in N .
\end{aligned}
$$

Assuming $V_{t+1}\left(X_{t+1}, w_{1: t}\right)$ is concave in $X_{t+1}$ (by induction), we prove $V_{t^{+}}$is a strictly concave optimization problem and furthermore, $V_{t}$ is strictly concave with respect to $X_{t}$ for every $w_{1: t} \in W_{1: t}$. For a give $w_{1: t}, C_{n, t}^{e}$ is strictly convex, $U_{t}$ is strictly concave and by assumption $V_{t+1}$ is concave. Since the domain is also concave, the problem is strictly concave optimization. The corresponding Lagrangian and KKT conditions are

$$
\begin{array}{r}
L_{t^{+}}\left(S_{t^{+}}\right)=U_{t}\left(\sum_{n \in N} e_{n, t}, w_{t}\right)-\sum_{n \in N} C_{n, t}^{e}\left(e_{n, t}, w_{t}\right)+\beta V_{t+1}\left(S_{t^{+}}\right) \\
+\sum_{n \in N} v_{n, t} e_{n, t}+\sum_{n \in N} \mu_{n, t}\left(x_{n, t^{+}}-e_{n, t}\right),
\end{array}
$$


and

$$
\begin{aligned}
\left.\frac{\partial U_{t}\left(\sum_{n \in N} e_{n, t}, w_{t}\right)}{\partial e_{n, t}}\right|_{e_{1: N, t}^{*}}-\left.\frac{\partial C_{n, t}^{e}\left(e_{n, t}, w_{t}\right)}{\partial e_{n, t}}\right|_{e_{1: N, t}^{*}} \\
+\left.\beta \frac{\partial V_{t+1}\left(S_{t^{+}}\right)}{\partial e_{n, t}}\right|_{e_{1: N, t}^{*}}+v_{n, t}^{*}-\mu_{n, t}^{*}=0, \\
v_{n, t}^{*} e_{n, t}^{*}=0, \\
\mu_{n, t}^{*}\left(x_{n, t^{+}}^{*}-e_{n, t}^{*}=0,\right. \\
v_{n, t}^{*} \geq 0, \quad \mu_{n, t}^{*} \geq 0,
\end{aligned}
$$

respectively.

Next, we prove that $V_{t^{+}}\left(S_{t^{+}}\right)$is concave in $X_{t^{+}}$. Consider $X_{t^{+}}^{1}, X_{t^{+}}^{2}, X_{t^{+}}^{3}=\lambda X_{t^{+}}^{1}+$ $(1-\lambda) X_{t^{+}}^{2}$, where $0 \leq \lambda \leq 1$ and their corresponding optimal production of electricity in Equation (A58), $e_{1: N, t}^{1}{ }^{*}, e_{1: N, t}^{2}{ }^{*}$ and $e_{1: N, t}^{3}{ }^{*}$. Consider $e_{1: N, t}^{3}=\lambda e_{1: N, t}^{1}{ }^{*}+(1-\lambda) e_{1: N, t}^{2}$. Since both $e_{1: N, t}^{1}{ }^{*}$ and $e_{1: N, t}^{2}{ }^{*}$ satisfy Equation (A59), $0 \leq e_{n, t}^{3} \leq \lambda x_{n, t^{+}}^{1}+(1-\lambda) x_{n, t^{+}}^{2}=x_{n, t^{+}}^{3}$ $\forall n \in N$. This means $e_{1: N, t}^{3}$ is a feasible point in the domain of optimization of $V_{t^{+}}$for $X_{t^{+}}^{3}$. In addition, since the objective function of function $V_{t^{+}}$is concave, we have

$$
\begin{aligned}
& \lambda V_{t^{+}}\left(X_{t}^{1}, w_{1: t}\right)+(1-\lambda) V_{t^{+}}\left(X_{t}^{2}, w_{1: t}\right)= \\
& \lambda\left[U_{t}\left(\sum_{n \in N} e_{n, t}^{1 *}, w_{t}\right)-\sum_{n \in N} C_{n, t}^{e}\left(e_{n, t}^{1 *}, w_{t}\right)+\beta V_{t+1}\left(X_{t^{+}}^{1}, w_{1: t}\right)\right] \\
& +(1-\lambda)\left[U_{t}\left(\sum_{n \in N} e_{n, t}^{2 *}, w_{t}\right)-\sum_{n \in N} C_{n, t}^{e}\left(e_{n, t}^{2 *}, w_{t}\right)+\beta V_{t+1}\left(X_{t^{+}}^{1}, w_{1: t}\right)\right] \\
& \leq U_{t}\left(\sum_{n \in N} e_{n, t}^{3}, w_{t}\right)-\sum_{n \in N} C_{n, t}^{e}\left(e_{n, t}^{3}, w_{t}\right)+\beta V_{t+1}\left(X_{t^{+}}^{1}, w_{1: t}\right) \\
& \leq U_{t}\left(\sum_{n \in N} e_{n, t}^{3 *}, w_{t}\right)-\sum_{n \in N} C_{n, t}^{e}\left(e_{n, t}^{3 *}, w_{t}\right)+\beta V_{t+1}\left(X_{t^{+}}^{1}, w_{1: t}\right)=V_{t^{+}}\left(X_{t^{+}}^{3}, w_{1: t}\right)
\end{aligned}
$$

This means $V_{n, t^{+}}$is concave in $X_{t^{+}}$.

Problem $Q_{t}^{3}$

The expansions at time $t$ are according to the following optimization problem, which we refer to by $Q_{t}^{3}$ (note that $\left(X_{t-1}+\Delta X_{t}, w_{1: t}\right)=S_{t^{+}}$).

$$
\begin{aligned}
V_{t}\left(S_{t}\right) & =\max _{\Delta x_{n, t}, n \in N}-\sum_{n} C_{n, t}^{x}\left(\Delta x_{n, t}, w_{t-1}\right)+E_{\mathbf{w}_{t}}\left[V_{t^{+}}\left(X_{t-1}+\Delta X_{t}, \mathbf{w}_{1: t}\right) \mid w_{1: t-1}\right] \\
\text { s.t. } & 0 \leq \Delta x_{n, t} \leq \overline{\Delta x}_{n, t} \\
& \sum_{n \in N} \Delta x_{n, t} \geq \Delta x_{t}
\end{aligned}
$$

Assuming that $V_{t^{+}}\left(X_{t-1}+\Delta X_{t}, \mathbf{w}_{1: t}\right)$ is concave with respect to $X_{t}=X_{t-1}+\Delta X_{t}$ (by induction), we prove $Q_{t}^{3}$ is a strictly concave optimization problem and furthermore, $V_{t}\left(S_{t}\right)$ is concave in $X_{t}$. First, to prove concavity of $V_{t}\left(S_{t}\right)$ with respect to $\Delta X_{t}$, define the function $B\left(\Delta X_{t}\right)=X_{t-1}+\Delta X_{t} . B\left(\Delta X_{t}\right)$ is concave and $V_{t}\left(S_{t}\right)$ is concave and nondecreasing in $X_{t}$, therefore, $V_{t^{+}}\left(B\left(\Delta X_{t}\right)\right)$ is concave in $\Delta X_{t}$ (See [91], Theorem 5.1) and $E_{\mathbf{w}_{t}}\left[V_{t^{+}}\left(X_{t-1}+\Delta X_{t}, \mathbf{w}_{1: t}\right) \mid w_{1: t-1}\right]$ is a weighted sum of concave functions, hence it is concave in $\Delta X_{t}$. Considering this, it is straightforward to see $V_{t}\left(S_{t}\right)$ is concave optimization with convex domain. The Lagrangian and the KKT conditions are

$$
\begin{gathered}
L_{t}\left(S_{t}\right)=-\sum_{n} C_{n, t}^{x}\left(\Delta x_{n, t}, w_{t-1}\right)+\beta E_{\mathbf{w}_{t}}\left[V_{t^{+}}\left(X_{t-1}+\Delta X_{t}, \mathbf{w}_{1: t}\right) \mid w_{1: t-1}\right]+ \\
\sum_{n \in N}\left(\alpha_{n, t} \Delta x_{n, t}+\beta_{n, t}\left(\overline{\Delta x}_{n, t}-\Delta x_{n, t}\right)\right)+\kappa_{t}\left(\sum_{n \in N} \Delta x_{n, t}-\underline{\Delta x}_{t}\right)
\end{gathered}
$$


and

$$
\begin{aligned}
& -\left.\frac{\partial C_{n, t}^{x}\left(\Delta x_{n, t}, w_{t-1}\right)}{\partial \Delta x_{n, t}}\right|_{\Delta X_{t}^{*}}+\beta E_{\mathbf{w}_{t}}\left[\left.\frac{\partial V_{t^{+}}\left(X_{t-1}+\Delta X_{t}, \mathbf{w}_{1: t}\right)}{\partial \Delta x_{n, t}}\right|_{\Delta X_{t}^{*}} \mid w_{1: t-1}\right] \\
& +\alpha_{n, t}^{*}-\beta_{n, t}^{*}+\kappa_{t}^{*}=0 \\
& \alpha_{n, t}^{*} \Delta x_{n, t}^{*}=0 \\
& \beta_{n, t}^{*}\left(\overline{\Delta x}_{n, t}-\Delta x_{n, t}^{*}\right)=0 \\
& \kappa_{t}^{*}\left(\sum_{n \in N} x_{n, t}^{*}-\underline{\Delta x_{t}}\right)=0 \\
& \alpha_{n, t}^{*} \geq 0, \quad \beta_{n, t}^{*} \geq 0, \kappa_{t}^{*} \geq 0
\end{aligned}
$$

respectively.

To prove $V_{t}\left(S_{t}\right)$ is concave in $X_{t}$ for a given $w_{1: t-1} \in W_{1: t-1}$, we use the first and the second order conditions. First, by envelope theorem,

$$
\frac{\partial V_{t}\left(S_{t}\right)}{\partial x_{n, t}}=\beta E_{\mathbf{w}_{t}}\left[\left.\frac{\partial V_{t^{+}}\left(X_{t-1}+\Delta X_{t}, \mathbf{w}_{1: t}\right)}{\partial x_{n, t}}\right|_{\Delta X_{t}^{*}} \mid w_{1: t-1}\right]
$$

which is non-negative by concavity of $V_{t^{+}}\left(X_{t-1}+\Delta X_{t}, \mathbf{w}_{1: t}\right)$ with respect to $X_{t-1}+\Delta X_{t}$ (first order condition). Second,

$$
\operatorname{Hessian}\left(V_{t}\left(S_{t}\right)\right)=\beta E_{\mathbf{w}_{t}}\left[\left.\operatorname{Hessian}\left(V_{t^{+}}\left(X_{t-1}+\Delta X_{t}, \mathbf{w}_{1: t}\right)\right)\right|_{\Delta X_{t}^{*}} \mid w_{1: t-1}\right]
$$

which is negative semidefinite by concavity of $V_{t^{+}}\left(X_{t-1}+\Delta X_{t}, \mathbf{w}_{1: t}\right)$ (second order condition). First and second order conditions prove concavity of $V_{t}\left(S_{t}\right)$.

Next we consider GenCos' best response. We fix the expansion and production strategies of all GenCos except GenCo $i$ to be Markovian. Then, GenCo $i$ 's best response problem is a constraint MDP where the constraints are on their capacity expansion and on the limits of their production at each instant of time. Therefore, there exists a best response for GenCo $i$ for production and expansion that is a Markovian strategy. Consequently if there exist SPNEs that are Markovian strategies, they will be determined by a set of $N$ coupled dynamic programs. The dynamic program for GenCo $n$ is the following.

Time $T+1$, GenCos' Best Response

$$
\tilde{V}_{n, T+1}\left(S_{T+1}\right)=\eta \tilde{x}_{n, T+1} .
$$

$\underline{\tilde{Q}_{n, t^{+}}^{3}}$

At the spot market of time $t^{+}$with $S_{t^{+}}$, GenCo's best response to other GenCos' production and price proposal $\tilde{m}_{-n, t^{+}}=\left(\hat{e}_{-n, t}, \hat{p}_{-n, t}\right)$ determined by their strategies $\hat{\gamma}_{-n, t}$ is

$$
\begin{aligned}
\tilde{V}_{n, t^{+}}\left(S_{t^{+}}\right)=\max _{\hat{e}_{n, t}, \hat{p}_{n, t}}-C_{n, t}^{e}\left(\hat{e}_{n, t}, w_{t}\right)+\hat{p}_{n+1, t} \hat{e}_{n, t}-\hat{p}_{n, t}^{-0.5} \gamma_{n, t}^{\text {elas. }}{ }_{n, t}^{2}+\beta \tilde{V}_{n, t+1}\left(S_{t+1}\right) \\
\text { s.t. } \quad 0 \leq \hat{e}_{n, t} \leq x_{n, t} \\
\quad 0 \leq \hat{p}_{n, t}
\end{aligned}
$$

$S_{t+1}$ is independent of $\hat{e}_{n, t}, \hat{p}_{n, t}$ conditioned on $S_{t}$ and $w_{t}$, and therefore, $\beta \tilde{V}_{n, t+1}\left(S_{t+1}\right)$ is independent of $\hat{e}_{n, t}, \hat{p}_{n, t}$. Therefore, it is straightforward to see $\tilde{V}_{n, t^{+}}$is concave optimization with respect to $\hat{e}_{n, t}, \hat{p}_{n, t}$ with convex domain. The Lagrangian and the KKT conditions are (note that $S_{t^{+}}=S_{t+1}$ ):

$$
\begin{aligned}
\tilde{L}_{n, t^{+}}\left(S_{t^{+}}\right) & =-C_{n, t}^{e}\left(\hat{e}_{n, t}, w_{t}\right)+\hat{p}_{n+1, t} \hat{e}_{n, t}-\hat{p}_{n, t}^{-0.5} \text { zlas. }_{n, t}^{2}+\beta \tilde{V}_{n, t+1}\left(S_{t^{+}}\right)+\hat{v}_{n, t} \hat{e}_{n, t} \\
& +\hat{\mu}_{n, t}\left(x_{n, t}-\hat{e}_{n, t}\right)+\hat{\theta}_{n, t} \hat{p}_{n, t}
\end{aligned}
$$


and

$$
\begin{aligned}
& -\left.\frac{\partial C_{n, t}^{e}}{\partial \hat{e}_{n, t}}\right|_{\hat{e}_{n, t}^{*}}+\hat{p}_{n+1, t}^{*}-\left.\frac{\partial \hat{p}_{n, t}^{-0.5} \text { zelas. }_{n, t}^{2}}{\partial \hat{e}_{n, t}}\right|_{\left(\tilde{m}_{-n, t}+\tilde{e}_{n, t}, \tilde{p}_{n, t}\right)} \\
& +\left.\beta \frac{\partial \tilde{V}_{n, t+1}\left(S_{t^{+}}\right)}{\partial \hat{e}_{n, t}}\right|_{\left(\tilde{m}_{-n, t}+\tilde{e}_{n, t}, \tilde{p}_{n, t}\right)}+\hat{v}_{n, t}^{*}-\hat{\mu}_{n, t}^{*}=0, \\
& -\left.\frac{\partial \hat{p}_{n, t}^{-0.5} \zeta^{\text {elas. }}{ }_{n, t}^{2}}{\partial \hat{p}_{n, t}}\right|_{\left(\tilde{m}_{-n, t}+, \tilde{e}_{n, t}^{*}, \tilde{p}_{n, t}^{*}\right)}+\left.\beta \frac{\partial \tilde{V}_{n, t+1}\left(S_{t+1}\right)}{\partial \hat{p}_{n, t}}\right|_{\left(\tilde{m}_{-n, t}+, \tilde{e}_{n, t}^{*}, \tilde{p}_{n, t}^{*}\right)}+\hat{\theta}_{n, t}^{*}=0, \\
& \hat{\theta}_{n, t}^{*} \hat{p}_{n, t}^{*}=0, \\
& \hat{\mu}_{n, t}^{*}\left(x_{n, t}-\hat{e}_{n, t}^{*}\right)=0, \\
& \hat{v}_{n, t}^{*} \hat{e}_{n, t}^{*}=0, \\
& \hat{v}_{n, t}^{*} \geq 0, \quad \hat{\mu}_{n, t}^{*} \geq 0, \quad \hat{\theta}_{n, t}^{*} \geq 0,
\end{aligned}
$$

respectively.

To prove that for every given $w_{1: t}, \tilde{V}_{n, t^{+}}\left(S_{t^{+}}\right)$is concave with respect to $x_{n, t^{+}}$, assume $\tilde{V}_{n, t+1}$ is concave in $x_{n, t+1}$ for every $w_{1: t} \in W_{1: t}$ (by induction). Now considering two different $x_{n, t^{+}}$and using the same approach used in the proof of concavity of $\hat{V}_{n, t^{+}}$, we can show $\tilde{V}_{n, t^{+}}\left(S_{t^{+}}\right)$is concave in $x_{n, t^{+}}$.

$\tilde{Q}_{n, t}^{3}$

At expansion epoch $t$ with $S_{t}$, GenCo $n$ 's best response to the other GenCos' expansion and price proposal $\tilde{m}_{-n, t}=\left(\Delta \tilde{x}_{-n, t}, \tilde{p}_{-n, t}\right)$ determined by their strategies $\tilde{\sigma}_{-n, t}$ is determined by

$$
\begin{aligned}
\tilde{V}_{n, t}\left(S_{t}\right)= & \max _{\Delta \tilde{x}_{n, t}, \tilde{p}_{n, t}}-C_{n, t}^{x}\left(\Delta \tilde{x}_{n, t}, w_{t-1}\right)+\tilde{p}_{n+1, t}^{*} \Delta \tilde{x}_{n, t}-\left(\tilde{p}_{n, t}-\tilde{p}_{n+1, t}^{*}\right)^{2}-2 \tilde{p}_{n, t} \zeta^{\text {inelas. }}{ }_{t}^{2} \\
& +\beta E_{\mathbf{w}_{t}}\left[\tilde{V}_{t^{+}, n}\left(X_{t-1}+\Delta \tilde{X}_{t}, \mathbf{w}_{1: t}\right) \mid w_{1: t-1}\right] \\
& 0 \leq \Delta \tilde{x}_{n, t} \leq \overline{\Delta x}_{n, t} \\
& \tilde{p}_{n, t} \geq 0 .
\end{aligned}
$$

We prove that the above optimization is a strictly concave optimization and furthermore, $\tilde{V}_{n, t}\left(S_{t}\right)$ is concave in $x_{n, t-1}$ for every $w_{1: t-1} \in W_{1: t-1}$. To do so, assume (by induction) that $\tilde{V}_{n, t^{+}}\left(X_{t-1}+\Delta \tilde{X}_{t}, w_{1: t}\right)$ is concave and increasing in $X_{t}+\Delta \tilde{X}_{t}$. Define function $B\left(\Delta \tilde{X}_{t}\right)=X_{t}+\Delta \tilde{X}_{t}$. Since $B\left(\Delta \tilde{X}_{t}\right)$ is affine, this means $\tilde{V}_{n, t^{+}}\left(X_{t}+\Delta \tilde{X}_{t}, w_{1: t}\right)$ is concave in $\Delta \tilde{x}_{n, t}$ (See [91], Theorem 5.1). Next, $E_{\mathbf{w}_{1: t}}\left[V_{n, t^{+}}\left(X_{t-1}+\Delta X_{t}, \mathbf{w}_{1: t}\right) \mid w_{1: t-1}\right]$ is a weighted sum of concave functions hence concave. Considering this, and the concavity

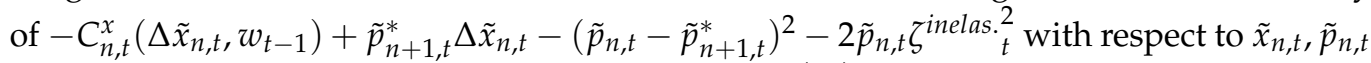
from Appendix A.1, it is straightforward to see $V_{n, t}\left(S_{t}\right)$ is a strictly concave optimization with convex domain. The Lagrangian and the KKT conditions are

$$
\begin{array}{r}
\tilde{L}_{n, t}\left(S_{t}\right)=-C_{n, t}^{x}\left(\Delta \tilde{x}_{n, t}, w_{t-1}\right)+\tilde{p}_{n+1, t}^{*} \Delta \tilde{x}_{n, t}-\left(\tilde{p}_{n, t}-\tilde{p}_{n+1, t}^{*}\right)^{2} \\
-2 \tilde{p}_{n, t} \zeta_{t}^{\text {inelas. }}{ }_{t}^{2}+\beta E_{\mathbf{w}_{t}}\left[\tilde{V}_{n, t^{+}}\left(X_{t-1}+\Delta \tilde{X}_{t}, \mathbf{w}_{1: t}\right) \mid w_{1: t-1}\right]+\tilde{\alpha}_{n, t} \Delta \tilde{x}_{n, t}+ \\
\tilde{\beta}_{n, t}\left(\overline{\Delta x} \bar{x}_{n, t}-\Delta \tilde{x}_{n, t}\right)+\tilde{\theta}_{n, t} \tilde{p}_{n, t},
\end{array}
$$

and 


$$
\begin{aligned}
& -\left.\frac{\partial C_{n, t}^{x}\left(\Delta \tilde{x}_{n, t}, w_{t-1}\right)}{\partial \Delta \tilde{x}_{n, t}}\right|_{\Delta \tilde{x}_{n, t}^{*}}+\left.\frac{\partial r_{n, t}^{\text {inelas. }}}{\partial \Delta \tilde{x}_{n, t}}\right|_{\left(\tilde{m}_{-n, t}, \Delta \tilde{x}_{n, t}^{*}, \tilde{p}_{n, t}^{*}\right)} \\
& +\beta E_{\mathbf{w}_{1: t}}\left[\left.\frac{\partial \tilde{V}_{n, t^{+}}\left(X_{t-1}+\Delta \tilde{X}_{t}, \mathbf{w}_{t}\right)}{\partial \Delta \tilde{x}_{n, t}}\right|_{\left(\tilde{m}_{-n, t}, \Delta \tilde{x}_{n, t}^{*}, \tilde{p}_{n, t}^{*}\right)} \mid w_{1: t-1}\right], \\
& +\tilde{\alpha}_{n, t}^{*}-\tilde{\beta}_{n, t}^{*}=0, \\
& \left.\frac{\partial r_{n, t}^{\text {inelas. }}}{\partial \tilde{p}_{n, t}}\right|_{\left(\tilde{m}_{-n, t} \Delta \tilde{x}_{n, t}^{*}, \tilde{e}_{n, t}^{*}\right)}+\beta E_{\mathbf{w}_{t}}\left[\left.\frac{\partial \tilde{V}_{n, t^{+}}\left(X_{t-1}+\Delta \tilde{X}_{t}, \mathbf{w}_{1: t}\right)}{\partial \tilde{p}_{n, t}}\right|_{\left(\tilde{m}_{-n, t}, \Delta \tilde{n}_{n, t}^{*} \tilde{r}_{n, t}^{*}\right)} \mid w_{1: t-1}\right], \\
& +\tilde{\alpha}_{n, t}^{*} \Delta \tilde{x}_{n, t}^{*}=0, \\
& \tilde{\beta}_{n, t}^{*}\left(\overline{\Delta x}_{n, t}-\Delta \tilde{x}_{n, t}^{*}\right)=0 \text {, } \\
& \tilde{\theta}_{n, t}^{*} \tilde{p}_{n, t}^{*}=0, \\
& \tilde{\alpha}_{n, t}^{*} \geq 0, \quad \tilde{\beta}_{n, t}^{*} \geq 0, \quad \tilde{\theta}_{n, t}^{*} \geq 0,
\end{aligned}
$$

respectively.

To prove $\tilde{V}_{n, t}\left(S_{t}\right)$ is concave in $x_{n, t}$, note that by induction, $\tilde{V}_{n, t^{+}}\left(X_{t-1}+\Delta \tilde{X}_{t}, \mathbf{w}_{t}\right)$ is concave and increasing in $X_{t^{+}}$. Defining the affine function $B\left(\Delta \tilde{X}_{t}\right)=X_{t-1}+\Delta \tilde{X}_{t}$ shows $\tilde{V}_{n, t^{+}}$is also concave in $\Delta \tilde{X}_{t}$ (See [91], Theorem 5.1). Now considering two different $X_{t}$ and using the same approach used in the proof of concavity of $\hat{V}_{n, t^{+}}$, we can show $\tilde{V}_{n, t}\left(S_{t}\right)$ is concave in $x_{n, t}$.

Finally, using the above concavity results, at the time $T+1$, by Lemma 2 there exists a Markovian Nash equilibrium. Furthermore, going backward, because the value functions at each time $t$ (and respectively $t^{+}$) are function of $S_{t}$ (respectively $S_{t^{+}}$), and concave as shown above, with the same proof of Lemma 2 there exist a Markovian NE at each time. The sequence of these NEs at each instant forms a Markovian SPNE.

Proof of Theorem 9. Part (i). We prove by construction. Assume $\hat{\sigma}_{n, t}^{*}$ and $\hat{\gamma}_{n, t}^{*}$ are the corresponding strategies for the dominant SPNE of the dynamic game induced by the mechanism proposed in Appendix B.2. By Lemma 3, these strategies are functions of $S_{t}$ and $S_{t^{+}}$, respectively. Define $\hat{e}_{n, t}^{*}\left(S_{t^{+}}\right)$to be GenCo $n^{\prime}$ s production at time $t^{+}$due to strategy $\tilde{\gamma}_{n, t}^{*}\left(S_{t^{+}}\right)$and $\Delta \tilde{x}_{n, t}^{*}\left(S_{t}\right)$ to be GenCo $n^{\prime}$ s expansion at time $t$ due to strategy $\tilde{\sigma}_{n, t}^{*}\left(S_{t}\right)$. Consider the following strategy for the planner.

$$
\begin{aligned}
\sigma_{n, t}^{*}\left(S_{t}\right) & =\Delta \tilde{x}_{n, t}^{*}\left(S_{t}\right) \\
\gamma_{n, t}^{*}\left(S_{t^{+}}\right) & =\hat{e}_{n, t}^{*}\left(S_{t^{+}}\right)
\end{aligned}
$$

Note that the capacity expansion is equivalent to an auction with inelastic demand; therefore, using the results of Theorem 3, Lemma 1, the KKT conditions of $\tilde{Q}_{n, t}^{3}$ and $\tilde{Q}_{n, t^{+}}^{3}$, Equations (A95)-(A100), Equations (A85)-(A90), respectively, and the generation and expansion strategies of Equations (A101) and (A102), the following parameters will satisfy the KKT conditions of $Q_{t}^{3}$, Equations (A72)-(A76), and the KKT conditions of $Q_{t^{+}}^{3}$, Equations (A62)-(A65). For all $t \in T$ and $n \in N$ put

$$
\begin{aligned}
v_{n, t}^{*} & =\tilde{v}_{n, t}^{*} \\
\mu_{n, t}^{*} & =\tilde{\mu}_{n, t}^{*} \\
\alpha_{n, t}^{*} & =\tilde{\alpha}_{n, t}^{*} \\
\beta_{n, t}^{*} & =\tilde{\beta}_{n, t}^{*}
\end{aligned}
$$

As a result, the energy production and expansion corresponding to the non-trivial SPNE are solutions of Problem (A43).

Part (ii) and (iii). To prove price efficiency and budget balanced in spot (or capacity) market of time $t^{+}$(time $t$ ), we model this market as a single elastic (or inelastic) auction presented in Section 3.2 and use the properties of these auctions presented in Section 3.5. To do 
this modeling, every GenCo's cost of generation (cost of expansion) is the sum of both the immediate utility from the spot (or capacity) market and the value of the future outcomes, $C_{n, t}^{e}\left(\hat{e}_{n, t}, w_{t}\right)-\beta \tilde{V}_{n, t+1}\left(S_{t}\right)$, (or $\left.C_{n, t}^{x}\left(\Delta \tilde{x}_{n, t}, w_{t-1}\right)-\beta E_{\mathbf{w}_{t}}\left[\tilde{V}_{t^{+}, n}\left(S_{t}, \Delta \tilde{X}_{t}, \tilde{p}_{t}, \mathbf{w}_{t}\right) \mid w_{1: t-1}\right]\right)$. This cost function is strictly convex and increasing and therefore the results of market clearance, Lemma 1, price efficiency, Theorem 1, and budget balance, Theorem 2, can be generalized for the spot (or capacity) market at time $t^{+}(t)$. This means Equations (A54)-(A56) hold.

Note that for the capacity market, the price at equilibrium is the dual variable of the long-term reliability constraint $\kappa_{t}^{*}$ (Equation (A71)). From Equation (A95), $\kappa_{t}^{*}$ represents the difference between the marginal cost of expansion and the marginal value of the future profits if $0<\Delta \tilde{x}_{n, t}<\overline{\Delta x}_{n, t}$ i.e., $\hat{\alpha}_{n, t}^{*}=0$ and $\hat{\beta}_{n, t}^{*}=0$.

Part (iv). To prove individual rationality, we should prove that, at each time instant $t$, by continuing participation in the mechanism, the GenCos' future utility is greater than or equal to zero (which is the utility they get by stopping to participate in the mechanism). We prove this by induction. We show that at time $T+1$ the GenCos' values are non-negative and then we show that if at any time $t$ (respectively $t^{+}$) it is weakly better for the GenCos to continue the game, then so is at $(t-1)^{+}$(respectively $t$ ).

First, note that at time $T+1, V_{n, T+1}, n \in N$ is non-negative by definition. Now assume at time $t+1$ it is individually rational for the GenCos to participate in the mechanism. Consider the generation epoch $t^{+}$. Since at the dominant SPNE, the firms act myopically in time $t^{+}$, by Theorem 4, their immediate revenue from the spot market is non-negative; therefore, the assumption that individual rationality holds at time $t+1$ results that it also holds at time $t^{+}$.

Next, assume the mechanism is individually rational starting from any $S_{t^{+}}$at time $t^{+}$. Consider time $t$. For the non-trivial NE, GenCo $n^{\prime}$ s utility from Theorem 9 Part (i) is $-C_{n, t}^{x}\left(\Delta \tilde{x}_{n, t}\right)+E\left\{\tilde{V}_{n, t}\left(X_{t-1}+\Delta \tilde{X}_{t}^{*}, w_{1: t}\right) \mid S_{t}\right\}+\hat{p}_{t}^{*} \Delta \tilde{x}_{n, t}^{*}$ where $\Delta \tilde{x}_{t}^{*}$ is the solution of $Q_{t}^{3}$; if $0=\Delta \tilde{x}_{n, t}^{*}$, then this utility is $E\left\{\tilde{V}_{n, t}\left(X_{t-1}+\Delta \tilde{X}_{t}^{*}, w_{1: t}\right) \mid S_{t}\right\}$ which is non-negative by assumption. If $0<\Delta \tilde{x}_{n, t}^{*}$ then

$$
\hat{p}_{t}^{*} \geq \frac{\partial\left(-C_{n, t}^{x}\left(\Delta \tilde{x}_{n, t}\right)+E\left\{\tilde{V}_{n, t}\left(X_{t-1}+\Delta \tilde{X}_{t}^{*}, w_{1: t} \mid S_{t}\right)\right\}\right)}{\partial \Delta \tilde{x}_{n, t}^{*}} ;
$$

since $-C_{n, t}^{x}\left(\Delta \tilde{x}_{n, t}\right)+E\left\{\tilde{V}_{n, t}\left(X_{t-1}+\Delta \tilde{X}_{t}^{*}, w_{1: t}\right) \mid S_{t}\right\}$ is strictly concave and the price $\hat{p}_{t}^{*}$ is the marginal of this utility, the participation utility at equilibrium $-C_{n, t}^{x}\left(\Delta \tilde{x}_{n, t}\right)+E\left\{\tilde{V}_{n, t}\left(X_{t-1}+\right.\right.$ $\left.\left.\Delta \tilde{X}_{t}^{*}, w_{1: t}\right) \mid S_{t}\right\}+\hat{p}_{t}^{*} \Delta \tilde{x}_{n, t}^{*}$ is non-negative. Therefore, it is individually rational to continue participation in the game at time $t$.

\section{References}

1. Vo, D.H. Sustainable Development Goals, Goal 7: Affordable and Clean Energy. Econ. Sociol. Available online: https: //www.un.org/sustainabledevelopment/energy/ (accessed on 9 August 2020).

2. Vo, D.H. Sustainable agriculture \& energy in the US: A link between ethanol production and the acreage for corn. Econ. Sociol. 2020, 13, 259-268.

3. Maroušek, J.; Struneckỳ, O.; Stehel, V. Biochar farming: Defining economically perspective applications. Clean Technol. Environ. Policy 2019, 21, 1389-1395. [CrossRef]

4. Mardoyan, A.; Braun, P. Analysis of Czech subsidies for solid biofuels. Int. J. Green Energy 2015, 12, 405-408. [CrossRef]

5. Maroušek, J.; Kolář, L.; Struneckỳ, O.; Kopeckỳ, M.; Bartoš, P.; Maroušková, A.; Cudlínová, E.; Konvalina, P.; Šoch, M.; Moudrỳ, J., Jr.; et al. Modified biochars present an economic challenge to phosphate management in wastewater treatment plants. J. Clean. Prod. 2020, 272, 123015. [CrossRef]

6. Joskow, P.; Tirole, J. Reliability and Competitive Electricity Markets. Rand J. Econ. 2007, 38, 60-84. [CrossRef]

7. Alizamir, S.; de Véricourt, F.; Sun, P. Efficient feed-in-tariff policies for renewable energy technologies. Oper. Res. 2016, 64, 52-66. [CrossRef]

8. Borenstein, S. The private and public economics of renewable electricity generation. J. Econ. Perspect. 2012, 26, 67-92. [CrossRef]

9. Acemoglu, D.; Akcigit, U.; Hanley, D.; Kerr, W. Transition to clean technology. J. Political Econ. 2016, 124, 52-104. [CrossRef] 
10. Hogan, W. A Cleaner Energy System: Renewable Energy and Electricity Market Design [In My View]. Power Energy Mag. IEEE 2015, 13. [CrossRef]

11. Garcia, A.; Shen, Z. Equilibrium capacity expansion under stochastic demand growth. Oper. Res. 2010, 58, 30-42. [CrossRef]

12. Murphy, F.H.; Smeers, Y. Generation capacity expansion in imperfectly competitive restructured electricity markets. Oper. Res. 2005, 53, 646-661. [CrossRef]

13. Dong, C.; Ng, C.T.; Cheng, T. Electricity time-of-use tariff with stochastic demand. Prod. Oper. Manag. 2017, 26, 64-79. [CrossRef]

14. Kim, J.H.; Powell, W.B. Optimal energy commitments with storage and intermittent supply. Oper. Res. 2011, 59, 1347-1360. [CrossRef]

15. Morales, J.M.; Zugno, M.; Pineda, S.; Pinson, P. Electricity market clearing with improved scheduling of stochastic production. Eur. J. Oper. Res. 2014, 235, 765-774. [CrossRef]

16. Pritchard, G.; Zakeri, G.; Philpott, A. A single-settlement, energy-only electric power market for unpredictable and intermittent participants. Oper. Res. 2010, 58, 1210-1219. [CrossRef]

17. Alizadeh, R.; Lund, P.D.; Soltanisehat, L. Outlook on biofuels in future studies: A systematic literature review. Renew. Sustain. Energy Rev. 2020, 134, 110326. [CrossRef]

18. Kök, A.G.; Shang, K.; Yücel, Ş. Impact of electricity pricing policies on renewable energy investments and carbon emissions. Manag. Sci. 2018, 64, 131-148. [CrossRef]

19. Pakurár, M.; Khan, M.A.; Benedek, A.; Oláh, J. The impact of green practices, cooperation and innovation on the performance of supply chains using statistical method of meta-analysis. J. Int. Stud. Vol. 2020, 13, 111-128. [CrossRef] [PubMed]

20. Maroušek, J. Study on agriculture decision-makers behavior on sustainable energy utilization. J. Agric. Environ. Ethics 2013, 26, 679-689. [CrossRef]

21. Muo, I.; Azeez, A.A. Green entrepreneurship: Literature review and agenda for future research. Int. J. Entrep. Knowl. 2019, 7, 17-29. [CrossRef]

22. Maroušek, J.; Myšková, K.; Žák, J. Managing environmental innovation: Case study on biorefinery concept. Rev. TÉCnica Fac. Ing. Univ. Del Zulia 2015, 38, 216-220.

23. Ritzenhofen, I.; Birge, J.R.; Spinler, S. Robustness of Renewable Energy Support Schemes Facing Uncertainty and Regulatory Ambiguity; Working Paper; Booth School of Business, University of Chicago: Chicago, IL, USA, 2014.

24. Liu, S.; Yang, Q.; Cai, H.; Yan, M.; Zhang, M.; Wu, D.; Xie, M. Market reform of Yunnan electricity in southwestern China: Practice, challenges and implications. Renew. Sustain. Energy Rev. 2019, 113, 109265. [CrossRef]

25. Kaleta, M. Aided design of market mechanisms for electricity clusters. Cent. Eur. J. Oper. Res. 2019, 28, 1-24. [CrossRef]

26. Palacio, S.M. Predicting collusive patterns in a liberalized electricity market with mandatory auctions of forward contracts. Energy Policy 2020, 139, 111311. [CrossRef]

27. Klemperer, P.D.; Meyer, M.A. Supply function equilibria in oligopoly under uncertainty. Econom. J. Econom. Soc. 1989, 57, 1243-1277. [CrossRef]

28. Green, R.J.; Newbery, D.M. Competition in the British electricity spot market. J. Political Econ. 1992, 100, 929-953. [CrossRef]

29. Anderson, E.J.; Hu, X. Finding supply function equilibria with asymmetric firms. Oper. Res. 2008, 56, 697-711. [CrossRef]

30. Singer, J.I. Double auctions across a constrained transmission line. Oper. Res. 2002, 50, 449-461. [CrossRef]

31. Banal-Estanol, A.; Micola, A.R. Composition of electricity generation portfolios, pivotal dynamics, and market prices. Manag. Sci. 2009, 55, 1813-1831. [CrossRef]

32. Peura, H.; Bunn, D.W. Dynamic pricing of peak production. Oper. Res. 2015, 63, 1262-1279. [CrossRef]

33. Wilson, R. Supply function equilibrium in a constrained transmission system. Oper. Res. 2008, 56, 369-382. [CrossRef]

34. Anderson, E.J. On the existence of supply function equilibria. Math. Program. 2013, 140, 323-349. [CrossRef]

35. Downward, A.; Zakeri, G.; Philpottt, A. On Cournot equilibria in electricity transmission networks. Oper. Res. 2010, 58, 1194-1209. [CrossRef]

36. Salant, D.; Stoddard, R. Auction Design for Capacity Markets; Working Paper; Auction Technologies: West Harwich, MA, USA, 2008.

37. Schummer, J.; Vohra, R.V. Auctions for procuring options. Oper. Res. 2003, 51, 41-51. [CrossRef]

38. Fabra, N.; Fehr, N.H.; Harbord, D. Designing electricity auctions. RAND J. Econ. 2006, 37, 23-46. [CrossRef]

39. FERC. FERC Docket Nos. ER10-787-000, EL10-50-000 and EL10-57-000, First Brief of ISO New England Inc.; Report; Federal Energy Regulatory Committee: Washington, DC, USA, 2012.

40. Cramton, P.; Ockenfels, A.; Stoft, S. Capacity market fundamentals. Econ. Energy Environ. Policy 2013, 2, 27-46. [CrossRef]

41. De Frutos, M.; Fabra, N. Endogenous capacities and price competition: The role of demand uncertainty. Int. J. Ind. Organ. 2011, 29, 399-411. [CrossRef]

42. Perakis, G.; Sun, W. Efficiency analysis of cournot competition in service industries with congestion. Manag. Sci. 2014, 60, 2684-2700. [CrossRef]

43. Bergemann, D.; Välimäki, J. Information acquisition and efficient mechanism design. Econometrica 2002, 70, 1007-1033. [CrossRef]

44. Ehrenmann, A.; Smeers, Y. Generation capacity expansion in a risky environment: A stochastic equilibrium analysis. Oper. Res. 2011, 59, 1332-1346. [CrossRef]

45. Joskow, P. Competitive Electricity Markets and Investment in New Generating Capacity; Working Paper; AEI-Brookings Joint Center: Washington, DC, USA, 2006.

46. Hogan, W. Electricity scarcity pricing through operating reserves. Econ. Energy Environ. Policy 2013, 2, 65-86. [CrossRef] 
47. Allaz, B.; Vila, J.L. Cournot competition, forward markets and efficiency. J. Econ. Theory 1993, 59, 1-16. [CrossRef]

48. Hogan, W. On an Energy only Electricity Market Design for Resource Adequacy; Working Paper; Harvard Kennedy School: Cambridge, MA, USA, 2005.

49. Henriot, A.; Glachant, J.M. Melting pots and salad bowls: The current debate on electricity market design for integration of intermittent RES. Util. Policy 2013, 27, 57-64. [CrossRef]

50. Hach, D.; Chyong, C.K.; Spinler, S. Capacity market design options: A dynamic capacity investment model and a GB case study. Eur. J. Oper. Res. 2016, 249, 691-705. [CrossRef]

51. Bushnell, J.B.; Ishii, J. An Equilibrium Model of Investment in Restructured Electricity Markets; Working Paper; Center for the Study of Energy Markets, University of California Berkeley: Berkeley, CA, USA, 2007.

52. Aflaki, S.; Netessine, S. Strategic investment in renewable energy sources: The effect of supply intermittency. Manuf. Serv. Oper. Manag. 2017, 19, 489-507. [CrossRef]

53. Rasouli, M.; Teneketzis, D. A methodology for Generation Expansion Planning for renewable energy economies. In Proceedings of the 2016 IEEE 55th Conference on Decision and Control (CDC), Las Vegas, NV, USA, 12-14 December 2016; pp. $1556-1563$.

54. Drake, D.F. Carbon tariffs: Effects in settings with technology choice and foreign production cost advantage. Manuf. Serv. Oper. Manag. 2018, 20, 667-686. [CrossRef]

55. Drake, D.F.; Kleindorfer, P.R.; Van Wassenhove, L.N. Technology choice and capacity portfolios under emissions regulation. Prod. Oper. Manag. 2016, 25, 1006-1025. [CrossRef]

56. Weitzman, M.L. Prices vs. quantities. Rev. Econ. Stud. 1974, 41, 477-491. [CrossRef]

57. Keohane, N.O. Cap and trade, rehabilitated: Using tradable permits to control US greenhouse gases. Rev. Environ. Econ. Policy 2009, 3, 42-62. [CrossRef]

58. Maskin, E. Nash equilibrium and welfare optimality. Rev. Econ. Stud. 1999, 66, 23-38. [CrossRef]

59. Dasgupta, P.; Hammond, P.; Maskin, E. The implementation of social choice rules: Some general results on incentive compatibility. Rev. Econ. Stud. 1979, 46, 185-216. [CrossRef]

60. Danilov, V. Implementation via Nash equilibria. Econom. J. Econom. Soc. 1992, 60, 43-56. [CrossRef]

61. Moulin, H. The Strategy of Social Choice; Elsevier: Amsterdam, The Netherlands, 1983.

62. Muller, E.; Satterthwaite, M.A. The equivalence of strong positive association and strategy-proofness. J. Econ. Theory 1977, 14, 412-418. [CrossRef]

63. Roberts, K. The characterization of implementable choice rules. Aggreg. Revel. Prefer. 1979, 12, 321-348.

64. Hurwicz, L. Outcome functions yielding Walrasian and Lindahl allocations at Nash equilibrium points. Rev. Econ. Stud. 1979, 46, 217-225. [CrossRef]

65. Moore, J.; Repullo, R. Nash implementation: A full characterization. Econometrica (1986-1998) 1990, 58, 1083. [CrossRef]

66. Dutta, B.; Sen, A. A necessary and sufficient condition for two-person Nash implementation. Rev. Econ. Stud. 1991, 58, 121-128. [CrossRef]

67. Maskin, E.; Sjöström, T. Implementation theory. Handb. Soc. Choice Welf. 2002, 1, 237-288.

68. Jackson, M.O. A crash course in implementation theory. Handb. Soc. Choice Welf. 2001, 18, 655-708. [CrossRef]

69. Sinha, A.; Anastasopoulos, A. A general mechanism design methodology for social utility maximization with linear constraints. ACM SIGMETRICS Perform. Eval. Rev. 2014, 42, 12-15. [CrossRef]

70. Farhadi, F.; Golestani, S.J.; Teneketzis, D. A surrogate optimization-based mechanism for resource allocation and routing in networks with strategic agents. IEEE Trans. Autom. Control 2019, 64, 464-479. [CrossRef]

71. Sharma, S.; Teneketzis, D. Local public good provisioning in networks: A Nash implementation mechanism. IEEE J. Sel. Areas Commun. 2012, 30, 2105-2116. [CrossRef]

72. Kuru, D.; Bayraktar, S. The effect of cyber-risk insurance to social welfare. J. Financ. Crime 2017, 24, 329-346. [CrossRef]

73. Rasouli, M.; Teneketzis, D. An efficient market design for electricity networks with strategic users possessing local information. IEEE Trans. Control. Netw. Syst. 2019, 6, 1038-1049. [CrossRef]

74. Moore, J.; Repullo, R. Subgame perfect implementation. Econom. J. Econom. Soc. 1988, 56, 1191-1220. [CrossRef]

75. Abreu, D.; Sen, A. Subgame perfect implementation: A necessary and almost sufficient condition. J. Econ. Theory 1990, 50, 285-299. [CrossRef]

76. Alizadeh, R.; Soltanisehat, L. Stay competitive in 2035: A scenario-based method to foresight in the design and manufacturing industry. Foresight 2020, 22, 309-330. [CrossRef]

77. Alizadeh, R.; Beiragh, R.G.; Soltanisehat, L.; Soltanzadeh, E.; Lund, P.D. Performance evaluation of complex electricity generation systems: A dynamic network-based data envelopment analysis approach. Energy Econ. 2020, 91, 104894. [CrossRef]

78. Jia, L.; Alizadeh, R.; Hao, J.; Wang, G.; Allen, J.K.; Mistree, F. A rule-based method for automated surrogate model selection. Adv. Eng. Inform. 2020, 45, 101123. [CrossRef]

79. Alizadeh, R.; Allen, J.K.; Mistree, F. Managing computational complexity using surrogate models: A critical review. Res. Eng. Des. 2020, 31, 275-298. [CrossRef]

80. Hnatyshyn, M. Decomposition analysis of the impact of economic growth on ammonia and nitrogen oxides emissions in the European Union. J. Int. Stud. 2018, 11, 201-209. [CrossRef]

81. Peters, E.; Kliestik, T.; Musa, H.; Durana, P. Product decision-making information systems, real-time big data analytics, and deep learning-enabled smart process planning in sustainable industry 4.0. J. Self-Gov. Manag. Econ. 2020, 8, 16-22. 
82. Valaskova, K.; Throne, O.; Kral, P.; Michalkova, L. Deep learning-enabled smart process planning in cyber-physical system-based manufacturing. J. Self-Gov. Manag. Econ. 2020, 8, 121-127.

83. Divényi, D.; Polgári, B.; Sleisz, Á.; Sőrés, P.; Raisz, D. Algorithm design for European electricity market clearing with joint allocation of energy and control reserves. Int. J. Electr. Power Energy Syst. 2019, 111, 269-285. [CrossRef]

84. Ventosa, M.; Baillo, A.; Ramos, A.; Rivier, M. Electricity market modeling trends. Energy Policy 2005, 33, 897-913. [CrossRef]

85. CAISO. California ISO Price Map. 2018. Available online: http://www.caiso.com/TodaysOutlook/Pages/Prices.aspx (accessed on 9 August 2020).

86. Cadwalader, M.; Gribik, P.; Hogan, W.; Pope, S. Extended LMP and Financial Transmission Rights; Working Paper; Harvard Kennedy School, Harvard University: Cambridge, MA, USA, 2010.

87. Ehrenmann, A.; Neuhoff, K. A comparison of electricity market designs in networks. Oper. Res. 2009, 57, 274-286. [CrossRef]

88. Wang, J.J.; Zender, J.F. Auctioning divisible goods. J. Econ. Theory 2002, 19, 673-705. [CrossRef]

89. Soltanisehat, L.; Alizadeh, R.; Hao, H.; Choo, K.K.R. Technical, Temporal, and Spatial Research Challenges and Opportunities in Blockchain-Based Healthcare: A Systematic Literature Review. IEEE Trans. Eng. Manag. 2020. [CrossRef]

90. Williams, J.; Alizadeh, R.; Allen, J.K.; Mistree, F. Using Network Partitioning to Design a Green Supply Chain. In International Design Engineering Technical Conferences and Computers and Information in Engineering Conference; American Society of Mechanical Engineers: New York, NY, USA, 2020; Volume 84010, p. V11BT11A050.

91. Rockafellar, R.T. Convex Analysis; Princeton University Press: Princeton, NJ, USA, 2015. 